\title{
Triarylaminium Radical Cation Promoted Coupling of Catharanthine with Vindoline: Diastereospecific Synthesis of Anhydrovinblastine and Reaction Scope
}

\author{
Byron A. Boon and Dale L. Boger* \\ Department of Chemistry and The Skaggs Institute of Chemical Biology, 10550 N. Torrey Pines Road, La Jolla, California \\ 92037 \\ *E-mail: boger@scripps.edu
}

\section{Supporting Information}

\begin{tabular}{cc}
\multicolumn{2}{c}{ Table of Contents } \\
Experimental Procedures & S2-S9 \\
References & S10 \\
${ }^{1}$ H NMR and ${ }^{13}$ C NMR Spectra & S11-S34 \\
X-Ray Data for 3'S-15 & S35-S36 \\
X-Ray Data for 3'R-16 $^{\prime}$ S37-S38
\end{tabular}




\section{Experimental Procedures}

General Information. All commercial compounds were used as received unless otherwise noted. Tris(4bromophenyl)ammoniumyl hexachloroantimonate (BAHA, 6) was purchased from Sigma Aldrich (lot \#MKBT9334V). Vindoline (3) was purchased from Acros Organics (lot \#A0380748). Catharanthine sulfate (4) was purchased from eNovation Chemicals (lot \#2016081101). 1,1,1-Trifluoroethanol (TFE) was purchased from Acros Organics (lot \#A0399038). 1,1,1,3,3,3-Hexafluoroisopropanol (HFIP) was purchased from Oakwood Chemical (lot \#003409I20E). All reactions were carried out in flame-dried glassware under an argon atmosphere, unless otherwise stated. Reactions were monitored using MilliporeSigma TLC plates $\left(0.25 \mathrm{~mm}, 60-\mathrm{F}_{254}\right)$ and were visualized using short-wave UV light as well as cerium ammonium molybdate stain. Preparative thin-layer chromatography (PTLC) was performed with MilliporeSigma plates $\left(0.5 \mathrm{~mm}, 60-\mathrm{F}_{254}\right)$. Column chromatography was performed using SiliCycle silica gel (40-63 micron) and reagent grade solvents. NMR spectra were recorded on a Brucker AV NEO 500 spectrometer ( $5 \mathrm{~mm}$ BBFO Smart Probe) or a Bruker AVIII HD 600 spectrometer (either a $5 \mathrm{~mm} \mathrm{CPDCH}$ CryoProbe or a $5 \mathrm{~mm} \mathrm{CPQCl}$ CryoProbe). Spectra recordings were calibrated to the solvent signal ( $\mathrm{CDCl}_{3} \delta=7.26 \mathrm{ppm}$ for ${ }^{1} \mathrm{H} \mathrm{NMR}$, and $\mathrm{CDCl}_{3} \delta=77.0 \mathrm{ppm}$ for ${ }^{13} \mathrm{C} \mathrm{NMR}$ ). Multiplicities are indicated by $s$ (singlet), d (doublet), t (triplet), q (quartet), $p$ (pentet), $m$ (multiplet), or b (broadened). Coupling constants $(J)(H, H)$ are given in Hz. IR spectra were recorded with a Nicolet 380 FTIR with an ATR attachment and selected peaks are reported in $\mathrm{cm}^{-1}$. High resolution mass spectral data was recorded on a Waters LC with a Waters G2-XS detector. Low resolution mass spectral data was recorded on an Agilent 1260 Infinity II LC with an InfinityLab LC/MSD detector. Optical rotations were recorded on a Rudolph Research Analytical Autopol III Automatic Polarimeter.

All new compounds are characterized by ${ }^{1} \mathrm{H} N M R,{ }^{13} \mathrm{C} N M R, I R, H R M S$, and optical rotation (S2, S3, 10e, 10f, 14c, 14d, 17). All known compounds prepared by the new methods detailed herein were characterized by ${ }^{1} \mathrm{H}$ NMR and LRMS and compared with literature reported data (with citations provided in the experimental which provides full characterization), all of which we had prepared previously and for which we have comparison samples. Starting material compounds and reagents $7,{ }^{1} \mathbf{8},{ }^{2} \mathbf{9},{ }^{2-3} \mathbf{1 1 a - 1 1 d},{ }^{4}$ and $\mathbf{1 2 - 1 3 ^ { 5 }}$ were prepared by previously established literature procedures and were identical in all respects with reported materials.

General Procedure A: Representative BAHA-Promoted Oxidative Coupling. A vial was charged with catharanthine sulfate $(4,8.7 \mathrm{mg}, 0.02 \mathrm{mmol})$, vindoline $(3,9.1 \mathrm{mg} 0.02 \mathrm{mmol})$, and a stir bar, then TFE $(0.38 \mathrm{~mL})$, aq $0.05 \mathrm{~N} \mathrm{HCl}(0.76 \mathrm{~mL})$, and BAHA $(6,18.0 \mathrm{mg}, 0.022 \mathrm{mmol})$ were added sequentially. The vessel was sealed with a screw cap with a Teflon septum and the reaction mixture was placed under inert atmosphere by addition of an argon balloon and the reaction was stirred for $2 \mathrm{~h}$ at $25{ }^{\circ} \mathrm{C}$. The mixture was cooled to $0{ }^{\circ} \mathrm{C}$, treated with $\mathrm{NaBH}_{4}(0.76 \mathrm{mg}, 0.02 \mathrm{mmol})$ in $\mathrm{H}_{2} \mathrm{O}(0.13 \mathrm{~mL})$, and stirred for $30 \mathrm{~min}$. The reaction was quenched by addition of aq $\mathrm{NH}_{4} \mathrm{OH}(28.0-30.0 \%)$ at $0{ }^{\circ} \mathrm{C}$, and the resulting mixture was extracted with $\mathrm{CH}_{2} \mathrm{Cl}_{2}(3 \mathrm{x})$. The organic extracts were combined, dried with $\mathrm{Na}_{2} \mathrm{SO}_{4}$, filtered, and concentrated in vacuo. Preparative thin-layer chromatography (PTLC, SiO $, 90: 9: 1 \quad \mathrm{EtOAc:MeOH:Et}{ }_{3} \mathrm{~N}$ ) gave $13.2 \mathrm{mg}$ (83\% yield) of anhydrovinblastine (5) as a white solid identical in all respects with authentic material. ${ }^{6-7}$

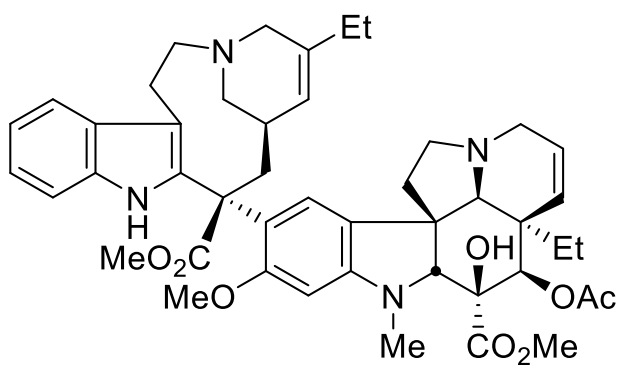

For anhydrovinblastine (5): ${ }^{1} \mathrm{H}$ NMR $\left(600 \mathrm{MHz}, \mathrm{CDCl}_{3}\right) \delta 9.81$ (bs, $\left.1 \mathrm{H}\right), 8.03(\mathrm{~s}, 1 \mathrm{H}), 7.51(\mathrm{~d}, J=7.8 \mathrm{~Hz}, 1 \mathrm{H}), 7.17$ (ddd, $J=$ 7.9, 6.7, $1.2 \mathrm{~Hz}, 1 \mathrm{H}), 7.13-7.08(\mathrm{~m}, 2 \mathrm{H}), 6.59(\mathrm{~s}, 1 \mathrm{H}), 6.12(\mathrm{~s}, 1 \mathrm{H}), 5.85$ (ddd, J = 10.2, 5.0, $1.6 \mathrm{~Hz}, 1 \mathrm{H}), 5.49(\mathrm{~d}, J=6.5 \mathrm{~Hz}$, $1 \mathrm{H}), 5.46(\mathrm{~s}, 1 \mathrm{H}), 5.30(\mathrm{dt}, J=10.2,2.1 \mathrm{~Hz}, 1 \mathrm{H}), 3.81(\mathrm{~s}, 3 \mathrm{H}), 3.79(\mathrm{~s}, 3 \mathrm{H}), 3.72(\mathrm{~s}, 1 \mathrm{H}), 3.61(\mathrm{~s}, 3 \mathrm{H}), 3.55(\mathrm{~d}, J=16.4 \mathrm{~Hz}, 1 \mathrm{H})$, $3.46-3.33(\mathrm{~m}, 3 \mathrm{H}), 3.32-3.17(\mathrm{~m}, 4 \mathrm{H}), 3.13-2.97(\mathrm{~m}, 2 \mathrm{H}), 2.82(\mathrm{dt}, J=16.1,2.1 \mathrm{~Hz}, 1 \mathrm{H}), 2.71(\mathrm{~s}, 3 \mathrm{H}), 2.66(\mathrm{~s}, 1 \mathrm{H}), 2.61$ 
$(\mathrm{d}, J=13.0 \mathrm{~Hz}, 1 \mathrm{H}), 2.50-2.36(\mathrm{~m}, 2 \mathrm{H}), 2.17-2.11(\mathrm{~m}, 1 \mathrm{H}), 2.10(\mathrm{~s}, 3 \mathrm{H}), 1.94(\mathrm{q}, J=7.5 \mathrm{~Hz}, 2 \mathrm{H}), 1.87-1.73(\mathrm{~m}, 2 \mathrm{H}), 1.42$ $-1.29(\mathrm{~m}, 2 \mathrm{H}), 0.99(\mathrm{t}, J=7.5 \mathrm{~Hz}, 3 \mathrm{H}), 0.80(\mathrm{t}, J=7.4 \mathrm{~Hz}, 3 \mathrm{H}) ;{ }^{13} \mathrm{CNMR}\left(150 \mathrm{MHz}, \mathrm{CDCl}_{3}\right) \delta 174.8,171.8,171.1,158.2,152.9$, 139.6, 135.1, 131.1, 130.1, 129.5, 124.7, 124.0, 123.7, 123.0, 122.5, 121.2, 119.1, 118.5, 117.0, 110.7, 94.4, 83.5, 79.8, 76.6, 65.6, 56.0, 55.6, 54.6, 53.4, 52.5, 52.4, 52.0, 50.4, 50.4, 45.9, 44.7, 42.8, 38.5, 34.5, 32.8, 31.0, 29.8, 27.9, 21.3, 12.3, 8.5; IR (neat, ATR) 3469, 2960, 2876, 1738, 1614, 1458, 1226, $1039 \mathrm{~cm}^{-1}$; HRMS (ESI-TOF): calcd [M+H] ${ }^{+}\left(\mathrm{C}_{46} \mathrm{H}_{57} \mathrm{~N}_{4} \mathrm{O}_{8}\right)$ 793.4176, found 793.4181; $[\alpha]_{D}^{23}+54\left(c 0.70, \mathrm{CHCl}_{3}\right)$.<smiles>COc1cccc(OCC(C)(C)O)c1</smiles>

1-(3-Methoxyphenoxy)-2-methylpropan-2-ol (S2). A stirred solution of methyl 2-(3-methoxyphenoxy)acetate ${ }^{8}$ (S1, 610 $\mathrm{mg}$, $3.11 \mathrm{mmol})$ in $\mathrm{Et}_{2} \mathrm{O}(9.1 \mathrm{~mL})$ was cooled to $0{ }^{\circ} \mathrm{C}$. $\mathrm{MeMgBr}\left(3 \mathrm{M}\right.$ in $\left.\mathrm{Et}_{2} \mathrm{O}\right)$ was added dropwise over $1 \mathrm{~h}$, and the mixture was stirred for $30 \mathrm{~min}$ at $0{ }^{\circ} \mathrm{C}$. The reaction mixture was poured into aq $2 \mathrm{~N} \mathrm{HCl}(8 \mathrm{~mL})$, and the mixture was extracted with EtOAc $(3 x)$. The organic layers were combined, dried with $\mathrm{Na}_{2} \mathrm{SO}_{4}$, filtered, and concentrated in vacuo. Column chromatography $\left(\mathrm{SiO}_{2}, 20 \%\right.$ EtOAc/hexanes) gave $515 \mathrm{mg}$ (84\%) of $\mathbf{S 2}$ as a clear light-yellow oil: ${ }^{1} \mathrm{H} \mathrm{NMR}\left(500 \mathrm{MHz}, \mathrm{CDCl}_{3}\right)$ $\delta 7.21(\mathrm{t}, J=8.2 \mathrm{~Hz}, 1 \mathrm{H}), 6.57-6.53(\mathrm{~m}, 2 \mathrm{H}), 6.51(\mathrm{t}, J=2.3 \mathrm{~Hz}, 1 \mathrm{H}), 3.82(\mathrm{~s}, 3 \mathrm{H}), 3.80(\mathrm{~s}, 2 \mathrm{H}), 2.26(\mathrm{bs}, 1 \mathrm{H}), 1.37(\mathrm{~s}, 6 \mathrm{H}) ;{ }^{13} \mathrm{C}$ NMR $\left(125 \mathrm{MHz}, \mathrm{CDCl}_{3}\right) \delta 160.9,160.0,129.9,106.8,106.7,101.1,76.0,70.1,55.3,26.2$; IR (neat, ATR) 3430, 2972, 2932, 1590, 1492, 1452, 1265, 1198, 1081, 1148, 1047, $922 \mathrm{~cm}^{-1}$; HRMS (ESI-TOF): calcd [M-OH] ${ }^{+}\left(\mathrm{C}_{12} \mathrm{H}_{15} \mathrm{O}_{2}\right)$ 179.1072, found 179.1072 .<smiles>COc1ccc2c(c1)OCC2(C)C</smiles>

6-Methoxy-3,3-dimethyl-2,3-dihydrobenzofuran (S3). A stirred solution of $\mathrm{P}_{2} \mathrm{O}_{5}(1.156 \mathrm{~g}, 8.15 \mathrm{mmol})$ in methanesulfonic acid $(10.6 \mathrm{~mL})$ was treated with $\mathbf{S 2}(457 \mathrm{mg}, 2.33 \mathrm{mmol})$ dropwise over $10 \mathrm{~min}$ with stirring at $25^{\circ} \mathrm{C}$. The reaction mixture was stirred $2 \mathrm{~h}$ before being poured into ice water and extracted with $\mathrm{Et}_{2} \mathrm{O}(3 \mathrm{x})$. The organic layers were combined, dried with $\mathrm{Na}_{2} \mathrm{SO}_{4}$, filtered, and concentrated in vacuo. Column chromatography ( $\mathrm{SiO}_{2}, 5 \%$ EtOAc/hexanes) gave $248 \mathrm{mg}(60 \%)$ of S3 as a clear colorless oil: ${ }^{1} \mathrm{H}$ NMR $\left(600 \mathrm{MHz}, \mathrm{CDCl}_{3}\right) 7.03(\mathrm{dt}, J=8.1,1.2 \mathrm{~Hz}, 1 \mathrm{H}), 6.40(\mathrm{dd}, J=8.1,2.3 \mathrm{~Hz}, 1 \mathrm{H}), 6.37(\mathrm{~d}, J$ $=2.3 \mathrm{~Hz}, 1 \mathrm{H}), 3.78(\mathrm{~s}, 3 \mathrm{H}), 2.96(\mathrm{~s}, 2 \mathrm{H}), 1.49$ (s, 7H); ${ }^{13} \mathrm{C} N M R\left(150 \mathrm{MHz}, \mathrm{CDCl}_{3}\right) \delta$ 160.4, 160.0, 125.1, 119.0, 105.5, 96.2, 87.7, 55.4, 42.2, 28.2; IR (neat, ATR) 2971, 2932, 1620, 1594, 1492, 1446, 1382, 1293, 1195, 1160, 1135, 1084, 1029, 952, $868 \mathrm{~cm}^{-1}$; HRMS (ESI-TOF): calcd [M+H] $]^{+}\left(\mathrm{C}_{12} \mathrm{H}_{15} \mathrm{O}_{2}\right)$ 179.1072, found 179.1068.<smiles>CCC1=C[C@@H](C[C@](C)(C(=O)OC)c2ccc(N(C)C)cc2OC)CCN1CC</smiles>

Compound 10a. Following general procedure A, catharanthine sulfate $(4,8.7 \mathrm{mg}, 0.02 \mathrm{mmol}), 3-\mathrm{methoxy}-\mathrm{N}, \mathrm{N}$ dimethylaniline $(3.0 \mathrm{mg}, 0.02 \mathrm{mmol})$, and BAHA $(6,18.0 \mathrm{mg}, 0.022 \mathrm{mmol})$ were combined and the reaction conducted in $\operatorname{TFE}(0.38 \mathrm{~mL})$ and aq $0.05 \mathrm{~N} \mathrm{HCl}(0.76 \mathrm{~mL})$. PTLC $\left(\mathrm{SiO}_{2}, 50 \%\right.$ EtOAc/hexanes) gave $1.5 \mathrm{mg}$ (15\% yield) of 10a identical in all respects with reported material: ${ }^{1} \mathrm{H}$ NMR $\left(600 \mathrm{MHz}, \mathrm{CDCl}_{3}\right) \delta 8.25(\mathrm{bs}, 1 \mathrm{H}), 7.50(\mathrm{~d}, J=8.0 \mathrm{~Hz}, 1 \mathrm{H}), 7.22(\mathrm{~d}, J=8.0 \mathrm{~Hz}, 1 \mathrm{H})$, $7.17(\mathrm{t}, J=7.4 \mathrm{~Hz}, 1 \mathrm{H}), 7.09(\mathrm{t}, J=7.4 \mathrm{~Hz}, 1 \mathrm{H}), 6.86(\mathrm{~d}, J=8.6 \mathrm{~Hz}, 1 \mathrm{H}), 6.31(\mathrm{~d}, J=2.5 \mathrm{~Hz}, 1 \mathrm{H}), 6.20(\mathrm{dd}, J=8.7,2.5 \mathrm{~Hz}, 1 \mathrm{H})$, $5.52(\mathrm{~d}, J=6.6 \mathrm{~Hz}, 1 \mathrm{H}), 3.83(\mathrm{~s}, 3 \mathrm{H}), 3.60(\mathrm{~s}, 3 \mathrm{H}), 3.55(\mathrm{~d}, J=16.0 \mathrm{~Hz}, 1 \mathrm{H}), 3.45(\mathrm{dd}, J=15.6,10.4 \mathrm{~Hz}, 1 \mathrm{H}), 3.40-3.30(\mathrm{~m}$, $2 \mathrm{H}), 3.26(\mathrm{pt}, J=12.2 \mathrm{~Hz}, 1 \mathrm{H}), 3.20(\mathrm{dd}, J=14.2,6.0 \mathrm{~Hz}, 1 \mathrm{H}), 3.13-3.02(\mathrm{~m}, 2 \mathrm{H}), 2.98(\mathrm{~s}, 6 \mathrm{H}), 2.63(\mathrm{~d}, J=12.9 \mathrm{~Hz}, 1 \mathrm{H}), 2.43$ 
(d, $J=15.3 \mathrm{~Hz}, 1 \mathrm{H}), 1.95(\mathrm{q}, J=7.5 \mathrm{~Hz}, 2 \mathrm{H}), 1.59-1.45(\mathrm{~m}, 1 \mathrm{H}), 1.00(\mathrm{t}, J=7.4 \mathrm{~Hz}, 3 \mathrm{H}) ;$ LRMS (ESI): calcd [M+H] ${ }^{+}\left(\mathrm{C}_{30} \mathrm{H}_{38} \mathrm{~N}_{3} \mathrm{O}_{3}\right)$ 488.3 , found 488.3 .<smiles>CCCc1ccccc1NC(=O)[C@](C)(C[C@@H]1C=C(CC)CN(CC)C1)c1ccc(N(C)C)cc1</smiles>

Compound 10b. Following general procedure A, catharanthine sulfate $(4,8.7 \mathrm{mg}, 0.02 \mathrm{mmol}), \mathrm{N}, \mathrm{N}$-dimethylaniline $(4.8$ $\mathrm{mg}, 0.04 \mathrm{mmol})$, and BAHA $(6,18.0 \mathrm{mg}, 0.022 \mathrm{mmol})$ were combined and the reaction conducted in HFIP $(0.38 \mathrm{~mL})$ and aq $0.05 \mathrm{~N} \mathrm{HCl}(0.76 \mathrm{~mL})$. PTLC ( $\mathrm{SiO}_{2}, 25 \%$ EtOAc/hexanes) gave $8.4 \mathrm{mg}$ (92\% yield) of $10 \mathrm{~b}$ as a white solid identical in all respects with reported material: ${ }^{1} \mathrm{H}$ NMR $\left(600 \mathrm{MHz}, \mathrm{CDCl}_{3}\right) \delta 7.78(\mathrm{bs}, 1 \mathrm{H}), 7.54(\mathrm{~d}, J=7.9 \mathrm{~Hz}, 1 \mathrm{H}), 7.20-7.11(\mathrm{~m}, 4 \mathrm{H})$, $7.09(\mathrm{t}, J=7.2 \mathrm{~Hz}, 1 \mathrm{H}), 6.66(\mathrm{~d}, J=8.5 \mathrm{~Hz}, 2 \mathrm{H}), 5.46(\mathrm{~d}, J=6.2 \mathrm{~Hz}, 1 \mathrm{H}), 3.57(\mathrm{~s}, 3 \mathrm{H}), 3.32-2.67(\mathrm{~m}, 5 \mathrm{H}), 3.21(\mathrm{dd}, J=14.7$, $7.4 \mathrm{~Hz}, 1 \mathrm{H}), 3.15(\mathrm{bd}, J=14.6 \mathrm{~Hz}, 1 \mathrm{H}), 2.95(\mathrm{~s}, 6 \mathrm{H}), 2.46-2.14(\mathrm{~m}, 2 \mathrm{H}), 2.10-1.70(\mathrm{~m}, 2 \mathrm{H}), 1.95(\mathrm{q}, J=7.5 \mathrm{~Hz}, 2 \mathrm{H}) 1.00(\mathrm{t}$, $J=7.5 \mathrm{~Hz}, 3 \mathrm{H})$; LRMS (ESI): calcd $[\mathrm{M}+\mathrm{H}]^{+}\left(\mathrm{C}_{29} \mathrm{H}_{36} \mathrm{~N}_{3} \mathrm{O}_{2}\right)$ 458.3, found 458.3 .<smiles>CCC1=C[C@H](C[C@](C)(c2ccc(OC)cc2OC)c2[nH]c3ccccc3c2CC)CN(CC)C1</smiles>

Compound 10c. Following general procedure A, catharanthine sulfate $(4,8.7 \mathrm{mg}, 0.02 \mathrm{mmol}), 1,3$-dimethoxybenzene ( 2.8 $\mathrm{mg}, 0.02 \mathrm{mmol})$, and BAHA $(6,18.0 \mathrm{mg}, 0.022 \mathrm{mmol})$ were combined and the reaction conducted in TFE $(0.38 \mathrm{~mL})$ and aq $0.05 \mathrm{~N} \mathrm{HCl}(0.76 \mathrm{~mL})$. PTLC ( $\mathrm{SiO}_{2}, 50 \%$ EtOAc/hexanes) gave $8.0 \mathrm{mg}(84 \%$ yield) of $10 \mathrm{c}$ as a white solid identical in all respects with reported material: ${ }^{1} \mathrm{H}$ NMR $\left(600 \mathrm{MHz}, \mathrm{CDCl}_{3}\right) \delta 8.19(\mathrm{~s}, 1 \mathrm{H}), 7.51(\mathrm{~d}, J=7.9 \mathrm{~Hz}, 1 \mathrm{H}), 7.24(\mathrm{~d}, J=8.1 \mathrm{~Hz}, 1 \mathrm{H})$, 7.18 (ddd, $J=8.1,6.9,1.2 \mathrm{~Hz}, 1 \mathrm{H}$ ), 7.10 (ddd, $J=8.0,6.9,1.1 \mathrm{~Hz}, 1 \mathrm{H}$ ), 6.96 (d, $J=8.6 \mathrm{~Hz}, 1 \mathrm{H}$ ), $6.54(\mathrm{~d}, J=2.5 \mathrm{~Hz}, 1 \mathrm{H}), 6.38$ (dd, J = 8.6, $2.5 \mathrm{~Hz}, 1 \mathrm{H}), 5.48(\mathrm{~d}, J=6.5 \mathrm{~Hz}, 1 \mathrm{H}), 3.82(\mathrm{~s}, 3 \mathrm{H}), 3.81(\mathrm{~s}, 3 \mathrm{H}), 3.60(\mathrm{~s}, 3 \mathrm{H}), 3.52(\mathrm{~d}, J=16.3 \mathrm{~Hz}, 1 \mathrm{H}), 3.41(\mathrm{ddd}, J$ $=15.3,9.6,2.2 \mathrm{~Hz}, 1 \mathrm{H}), 3.34-3.27(\mathrm{~m}, 2 \mathrm{H}), 3.25-3.16(\mathrm{~m}, 2 \mathrm{H}), 3.09(\mathrm{dd}, J=15.5,12.4 \mathrm{~Hz}, 1 \mathrm{H}), 3.04(\mathrm{dd}, J=15.8,6.0 \mathrm{~Hz}$, $1 \mathrm{H}), 2.59(\mathrm{~d}, J=13.0 \mathrm{~Hz}, 1 \mathrm{H}), 2.44(\mathrm{~d}, J=14.9 \mathrm{~Hz}, 1 \mathrm{H}), 1.94(\mathrm{q}, J=7.5 \mathrm{~Hz}, 2 \mathrm{H}), 1.45-1.37(\mathrm{~m}, 1 \mathrm{H}), 1.00(\mathrm{t}, J=7.4 \mathrm{~Hz}, 3 \mathrm{H})$; LRMS (ESI): calcd [M+H] ${ }^{+}\left(\mathrm{C}_{29} \mathrm{H}_{35} \mathrm{~N}_{2} \mathrm{O}_{4}\right)$ 475.3, found 475.3.<smiles>CCCN1CC(CC)=C[C@H](C[C@](C)(C(=O)Nc2ccccc2OC)c2cc3c(cc2OC)OCC3(C)C)C1</smiles>

Compound 10e. Following general procedure A, catharanthine sulfate $(4,8.7 \mathrm{mg}, 0.02 \mathrm{mmol}), \mathbf{S 3}(3.6 \mathrm{mg}, 0.02 \mathrm{mmol})$, and BAHA $(6,18.0 \mathrm{mg}, 0.022 \mathrm{mmol})$ were combined and the reaction conducted in TFE $(0.38 \mathrm{~mL})$ and aq $0.05 \mathrm{~N} \mathrm{HCl}(0.76 \mathrm{~mL})$. PTLC ( $\mathrm{SiO}_{2}, 90: 9: 1$ hexanes/EtOAc/Et $\left.{ }_{3} \mathrm{~N}\right)$ gave $7.6 \mathrm{mg}\left(74 \%\right.$ yield) of $10 \mathrm{e}$ as a white solid: ${ }^{1} \mathrm{H} \mathrm{NMR}\left(600 \mathrm{MHz}, \mathrm{CDCl}_{3}\right) \delta 8.28$ $(\mathrm{s}, 1 \mathrm{H}), 7.54(\mathrm{~d}, J=7.9 \mathrm{~Hz}, 1 \mathrm{H}), 7.30(\mathrm{~d}, J=8.0 \mathrm{~Hz}, 1 \mathrm{H}), 7.21$ (ddd, $J=8.1,6.9,1.1 \mathrm{~Hz}, 1 \mathrm{H}), 7.13$ (ddd, $J=8.0,7.0,1.1 \mathrm{~Hz}$, $1 \mathrm{H}), 6.80(\mathrm{~s}, 1 \mathrm{H}), 6.44(\mathrm{~s}, 1 \mathrm{H}), 5.53(\mathrm{~d}, J=6.5 \mathrm{~Hz}, 1 \mathrm{H}), 3.80(\mathrm{~s}, 3 \mathrm{H}), 3.63(\mathrm{~s}, 3 \mathrm{H}), 3.55(\mathrm{~d}, J=16.4 \mathrm{~Hz}, 1 \mathrm{H}), 3.43$ (ddd, $J=15.3$, 9.5, $2.2 \mathrm{~Hz}, 1 \mathrm{H}), 3.36-3.29(\mathrm{~m}, 2 \mathrm{H}), 3.28-3.17(\mathrm{~m}, 2 \mathrm{H}), 3.17-3.02(\mathrm{~m}, 2 \mathrm{H}), 2.91(\mathrm{~d}, J=15.3 \mathrm{~Hz}, 1 \mathrm{H}), 2.83(\mathrm{~d}, J=15.1 \mathrm{~Hz}$, $1 \mathrm{H}), 2.62(\mathrm{~d}, J=13.9 \mathrm{~Hz}, 1 \mathrm{H}), 2.46(\mathrm{~d}, J=12.7 \mathrm{~Hz}, 1 \mathrm{H}), 1.97(\mathrm{q}, J=7.5 \mathrm{~Hz}, 2 \mathrm{H}), 1.52(\mathrm{~s}, 3 \mathrm{H}), 1.46(\mathrm{~s}, 3 \mathrm{H}), 1.03(\mathrm{t}, J=7.5 \mathrm{~Hz}$, 
$3 \mathrm{H}) ;{ }^{13} \mathrm{C} \mathrm{NMR}\left(150 \mathrm{MHz}, \mathrm{CDCl}_{3}\right) \delta 174.4,158.8,156.7,134.4,130.4,128.6,125.7,123.7,121.9,121.4,118.5,118.0,117.4$, 110.0, 94.4, 87.6, 55.4, 55.0, 54.1, 51.9, 51.3, 45.6, 45.5, 41.9, 33.7, 32.2, 29.2, 27.9, 27.8, 27.4, 11.8 (one C, absent); IR (neat, ATR) 3434, 3050, 2967, 2928, 2851, 1726, 1458, 1285, 1265, 1196, 1131, 1091, 778, $733 \mathrm{~cm}^{-1}$; HRMS (ESI-TOF): calcd $[\mathrm{M}+\mathrm{H}]^{+}\left(\mathrm{C}_{32} \mathrm{H}_{39} \mathrm{~N}_{2} \mathrm{O}_{4}\right) 515.2910$, found 515.2915; $[\alpha]_{D}{ }^{25}+101$ (c 1.56, $\left.\mathrm{CHCl}_{3}\right)$.

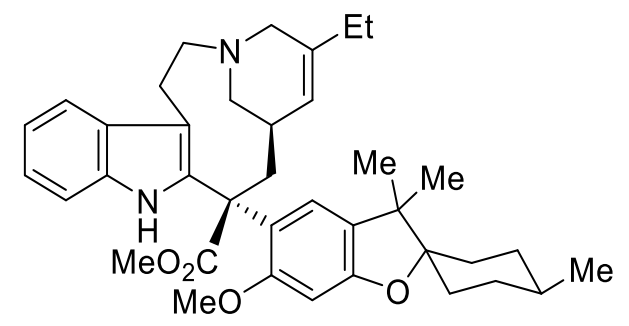

Compound 10f. Following general procedure $A$, catharanthine sulfate $(4,8.7 \mathrm{mg}, 0.02 \mathrm{mmol})$, the aromatic-coupling partner $(5.2 \mathrm{mg}, 0.02 \mathrm{mmol}),{ }^{10}$ and BAHA $(6,18.0 \mathrm{mg}, 0.022 \mathrm{mmol})$ were combined and the reaction conducted in HFIP $(0.38 \mathrm{~mL})$ and aq $0.05 \mathrm{~N} \mathrm{HCl}(0.76 \mathrm{~mL})$. PTLC $\left(\mathrm{SiO}_{2}, 50 \%\right.$ EtOAc/hexanes) gave $8.2 \mathrm{mg}$ (68\% yield) of $10 \mathrm{Of}$ as a white solid: ${ }^{1} \mathrm{H}$ $\operatorname{NMR}\left(600 \mathrm{MHz}, \mathrm{CDCl}_{3}\right) \delta 8.23(\mathrm{~s}, 1 \mathrm{H}), 7.52(\mathrm{~d}, J=7.9 \mathrm{~Hz}, 1 \mathrm{H}), 7.27-7.24(\mathrm{~m}, 1 \mathrm{H}), 7.19(\mathrm{t}, J=7.5 \mathrm{~Hz}, 1 \mathrm{H}), 7.12(\mathrm{t}, J=7.3 \mathrm{~Hz}$, $1 \mathrm{H}), 6.64(\mathrm{~s}, 1 \mathrm{H}), 6.48(\mathrm{~s}, 1 \mathrm{H}), 5.51(\mathrm{~s}, 1 \mathrm{H}), 3.77(\mathrm{~s}, 3 \mathrm{H}), 3.60(\mathrm{~s}, 3 \mathrm{H}), 3.56(\mathrm{~d}, J=16.1 \mathrm{~Hz}, 1 \mathrm{H}), 3.49-3.15(\mathrm{~m}, 2 \mathrm{H}), 3.14-$ $3.01(\mathrm{~m}, 6 \mathrm{H}), 2.61(\mathrm{~d}, J=13.4 \mathrm{~Hz}, 1 \mathrm{H}), 2.38(\mathrm{~d}, J=15.3 \mathrm{~Hz}, 1 \mathrm{H}), 2.01-1.89(\mathrm{~m}, 3 \mathrm{H}), 1.79(\mathrm{dd}, J=13.3,3.0 \mathrm{~Hz}, 1 \mathrm{H}), 1.66-$ $1.54(\mathrm{~m}, 3 \mathrm{H}), 1.49-1.32(\mathrm{~m}, 6 \mathrm{H}), 1.06(\mathrm{~s}, 3 \mathrm{H}), 1.01(\mathrm{t}, J=7.5 \mathrm{~Hz}, 3 \mathrm{H}), 0.96(\mathrm{~d}, J=5.6 \mathrm{~Hz}, 3 \mathrm{H}), 0.92(\mathrm{~s}, 1 \mathrm{H}) ;{ }^{13} \mathrm{C} \mathrm{NMR}(150$ $\left.\mathrm{MHz}, \mathrm{CDCl}_{3}\right) \delta 174.9,171.3,157.7,156.9,135.1,131.2,129.7,129.2,124.4,123.8,122.4,119.1,118.5,116.8,110.5,95.6$, 93.0, 60.5, 56.0, 55.7, 54.5, 52.5, 52.0, 46.1, 45.9, 34.6, 32.0, 31.1, 30.9, 30.8, 29.8, 28.0, 24.2, 24.1, 22.4, 21.2, 14.3, 12.3; IR (neat, ATR) 3466, 3394, 2950, 2922, 2854, 1730, 1617, 1488, 1458, 1221, 1193, 1161, 1058, $1007 \mathrm{~cm}^{-1}$; HRMS (ESI-TOF): calcd $[\mathrm{M}+\mathrm{H}]^{+}\left(\mathrm{C}_{38} \mathrm{H}_{49} \mathrm{~N}_{2} \mathrm{O}_{4}\right)$ 597.3692, found 597.3682; $[\alpha]_{D}{ }^{25}+75$ (c 0.27, $\left.\mathrm{CHCl}_{3}\right)$.<smiles>CCC1=C[C@@H](C[C@](C)(C(=O)OC)c2c[nH]c3ccccc23)CN(CCCc2ccccc2OC)C1</smiles>

Compound 10g. Following general procedure $A$, catharanthine sulfate $(4,8.7 \mathrm{mg}, 0.02 \mathrm{mmol})$, indole $(2.3 \mathrm{mg}, 0.02 \mathrm{mmol})$, and BAHA $(6,18.0 \mathrm{mg}, 0.022 \mathrm{mmol})$ were combined and the reaction conducted in HFIP $(0.38 \mathrm{~mL})$ and aq $0.05 \mathrm{~N} \mathrm{HCl}(0.76$ $\mathrm{mL}$ ). Note: the reaction mixture was quenched with saturated aq $\mathrm{Na}_{2} \mathrm{~S}_{2} \mathrm{O}_{3}(2.5 \mathrm{~mL})$ prior to the $\mathrm{NH}_{4} \mathrm{OH}$ quench. $\mathrm{PTLC}\left(\mathrm{SiO}_{2}\right.$, $50 \% \mathrm{EtOAc} /$ hexanes) gave $6.3 \mathrm{mg}\left(69 \%\right.$ yield) of $10 \mathrm{~g}$ as a white solid identical in all respects with reported material: ${ }^{1} \mathrm{H}$ $\operatorname{NMR}\left(600 \mathrm{MHz}, \mathrm{CDCl}_{3}\right) \delta 8.27$ (bs, 1H), 7.91 (bs, $\left.1 \mathrm{H}\right), 7.62-7.55(\mathrm{~m}, 1 \mathrm{H}), 7.33(\mathrm{~d}, J=8.1 \mathrm{~Hz}, 1 \mathrm{H}), 7.26(\mathrm{~d}, J=8.3 \mathrm{~Hz}, 1 \mathrm{H})$, $7.16-7.04(\mathrm{~m}, 4 \mathrm{H}), 6.92(\mathrm{t}, J=7.3 \mathrm{~Hz}, 1 \mathrm{H}), 5.45(\mathrm{~d}, J=6.0 \mathrm{~Hz}, 1 \mathrm{H}), 3.75-3.65(\mathrm{~m}, 1 \mathrm{H}), 3.53(\mathrm{~s}, 3 \mathrm{H}), 3.26-3.11(\mathrm{~m}, 3 \mathrm{H})$, $3.05(\mathrm{~d}, J=14.3 \mathrm{~Hz}, 1 \mathrm{H}), 2.97-2.66(\mathrm{~m}, 3 \mathrm{H}), 2.48(\mathrm{dd}, J=14.5,6.3 \mathrm{~Hz}, 1 \mathrm{H}), 2.27-2.18(\mathrm{~m}, 1 \mathrm{H}), 2.04-1.91(\mathrm{~m}, 2 \mathrm{H}), 1.01$ $(\mathrm{t}, J=7.5 \mathrm{~Hz}, 3 \mathrm{H})$; LRMS (ESI): calcd $[\mathrm{M}+\mathrm{H}]^{+}\left(\mathrm{C}_{29} \mathrm{H}_{32} \mathrm{~N}_{3} \mathrm{O}_{2}\right) 454.3$, found 454.3.<smiles>CCC1=C[C@@H](C[C@](C)(C(C)=O)c2ccc[nH]2)CN(CCc2ccccc2NC(C)C)C1</smiles>

Compound 10h. Following general procedure $A$, catharanthine sulfate $(4,8.7 \mathrm{mg}, 0.02 \mathrm{mmol})$, pyrrole $(1.3 \mathrm{mg}, 0.02 \mathrm{mmol})$, and BAHA $(6,18.0 \mathrm{mg}, 0.022 \mathrm{mmol})$ were combined and the reaction conducted in TFE $(0.38 \mathrm{~mL})$ and aq $0.05 \mathrm{~N} \mathrm{HCl}(0.76$ 
$\mathrm{mL})$. PTLC ( $\mathrm{SiO}_{2}, 25 \%$ EtOAc/hexanes) gave $3.8 \mathrm{mg}$ (47\% yield) of $10 \mathrm{~h}$ as a white solid identical in all respects with reported material: ${ }^{1} \mathrm{H}$ NMR $\left(600 \mathrm{MHz}, \mathrm{CDCl}_{3}\right) \delta 8.26$ (bs, $\left.1 \mathrm{H}\right), 7.57(\mathrm{~d}, J=7.8 \mathrm{~Hz}, 1 \mathrm{H}), 7.45(\mathrm{bs}, 1 \mathrm{H}), 7.15$ (d, J = 7.9 Hz, $\left.1 \mathrm{H}\right), 7.11(\mathrm{t}, J$ $=7.2 \mathrm{~Hz}, 1 \mathrm{H}), 7.07(\mathrm{t}, J=7.1 \mathrm{~Hz}, 1 \mathrm{H}), 6.66(\mathrm{~s}, 1 \mathrm{H}), 6.37(\mathrm{~s}, 1 \mathrm{H}), 6.16(\mathrm{dd}, J=2.9,2.9 \mathrm{~Hz}, 1 \mathrm{H}), 5.46(\mathrm{bs}, 1 \mathrm{H}), 3.58-3.50(\mathrm{~m}$, $1 \mathrm{H}), 3.57(\mathrm{~s}, 3 \mathrm{H}), 3.06-2.95(\mathrm{~m}, 3 \mathrm{H}), 2.91(\mathrm{~d}, J=13.8 \mathrm{~Hz}, 1 \mathrm{H}), 2.67(\mathrm{dd}, J=13.0,5.4 \mathrm{~Hz}, 1 \mathrm{H}), 2.52(\mathrm{dd}, J=14.4,8.2 \mathrm{~Hz}, 1 \mathrm{H})$, $2.39(\mathrm{t}, J=12.5 \mathrm{~Hz}, 1 \mathrm{H}), 2.36-2.27(\mathrm{~m}, 2 \mathrm{H}), 2.08-1.89(\mathrm{~m}, 3 \mathrm{H}), 1.04(\mathrm{t}, J=7.5 \mathrm{~Hz}, 3 \mathrm{H})$. LRMS (ESI): calcd [M+H] $\left(\mathrm{C}_{25} \mathrm{H}_{30} \mathrm{~N}_{3} \mathrm{O}_{2}\right)$ 403.2, found 403.2.

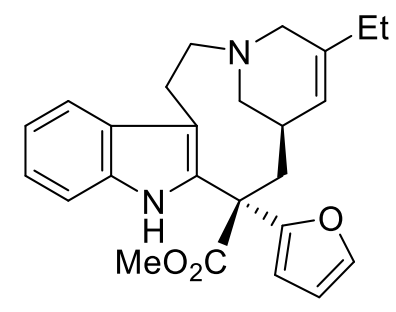

Compound 10i. Following general procedure $A$, catharanthine sulfate $(4,8.7 \mathrm{mg}, 0.02 \mathrm{mmol})$, furan $(4.1 \mathrm{mg}, 0.06 \mathrm{mmol})$, and BAHA $(6,18.0 \mathrm{mg}, 0.022 \mathrm{mmol})$ were combined and the reaction conducted in TFE $(0.38 \mathrm{~mL})$ and aq $0.05 \mathrm{~N} \mathrm{HCl}(0.76$ $\mathrm{mL}$ ). PTLC ( $\mathrm{SiO}_{2}, 25 \% \mathrm{EtOAc} /$ hexanes) gave $3.2 \mathrm{mg}$ (40\% yield) of $\mathbf{1 0 i}$ as a white solid identical in all respects with reported material: ${ }^{1} \mathrm{H}$ NMR $\left(500 \mathrm{MHz}, \mathrm{CDCl}_{3}\right) \delta 7.93(\mathrm{~s}, 1 \mathrm{H}), 7.55(\mathrm{~d}, J=7.9 \mathrm{~Hz}, 1 \mathrm{H}), 7.41(\mathrm{dd}, J=1.8,0.9 \mathrm{~Hz}, 1 \mathrm{H}), 7.23(\mathrm{~d}, J=8.0 \mathrm{~Hz}$, $1 \mathrm{H}$ ), 7.15 (ddd, $J=8.0,7.0,1.2 \mathrm{~Hz}, 1 \mathrm{H}$ ), 7.09 (ddd, $J=8.0,6.9,1.1 \mathrm{~Hz}, 1 \mathrm{H}$ ), 6.38 (dd, $J=3.3,1.8 \mathrm{~Hz}, 1 \mathrm{H}$ ), 6.37 (dd, $J=3.3$, $0.9 \mathrm{~Hz}, 1 \mathrm{H}), 5.47(\mathrm{~d}, J=6.0 \mathrm{~Hz}, 1 \mathrm{H}), 3.64(\mathrm{~s}, 3 \mathrm{H}), 3.53-3.44(\mathrm{~m}, 1 \mathrm{H}), 3.21(\mathrm{~d}, J=15.6 \mathrm{~Hz}, 1 \mathrm{H}), 3.15-3.04(\mathrm{~m}, 2 \mathrm{H}), 2.98(\mathrm{dt}$, $J=14.3,2.6 \mathrm{~Hz}, 1 \mathrm{H}), 2.91-2.82(\mathrm{~m}, 1 \mathrm{H}), 2.79-2.67(\mathrm{~m}, 2 \mathrm{H}), 2.43(\mathrm{dd}, J=14.7,5.8 \mathrm{~Hz}, 1 \mathrm{H}), 2.25(\mathrm{~d}, J=11.7 \mathrm{~Hz}, 1 \mathrm{H}), 2.04$ $-1.88(\mathrm{~m}, 3 \mathrm{H}), 1.01(\mathrm{t}, J=7.5 \mathrm{~Hz}, 3 \mathrm{H})$; LRMS (ESI): calcd [M+H] ${ }^{+}\left(\mathrm{C}_{25} \mathrm{H}_{28} \mathrm{~N}_{2} \mathrm{O}_{3}\right)$ 405.2, found 405.2.

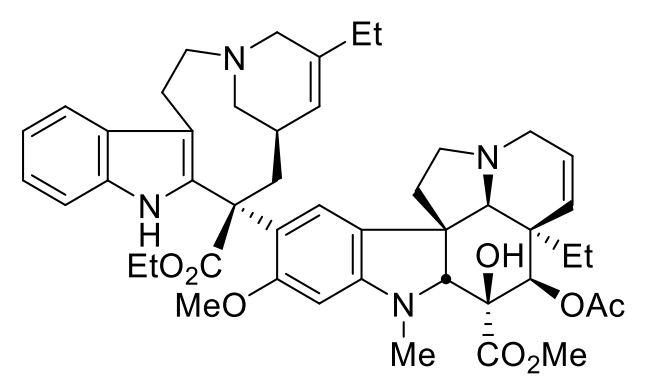

Compound 14a. Following general procedure A, 11a $(7.0 \mathrm{mg}, 0.02 \mathrm{mmol})$, vindoline $(3,9.1 \mathrm{mg}, 0.02 \mathrm{mmol})$, and BAHA (6, $18.0 \mathrm{mg}, 0.022 \mathrm{mmol}$ ) were combined and the reaction conducted in TFE $(0.38 \mathrm{~mL})$ and aq $0.05 \mathrm{~N} \mathrm{HCl}(0.76 \mathrm{~mL})$. PTLC $\left(\mathrm{SiO}_{2}, 90: 9: 1 \mathrm{EtOAc}: \mathrm{MeOH}: \mathrm{Et}_{3} \mathrm{~N}\right)$ gave $9.9 \mathrm{mg}(61 \%$ yield) of $14 \mathrm{a}$ as a white solid identical in all respects with reported material: ${ }^{1} \mathrm{H}$ NMR $\left(600 \mathrm{MHz}, \mathrm{CDCl}_{3}\right) \delta 9.85(\mathrm{~s}, 1 \mathrm{H}), 8.06(\mathrm{~s}, 1 \mathrm{H}), 7.52(\mathrm{~d}, J=8.1 \mathrm{~Hz}, 1 \mathrm{H}), 7.19-7.13(\mathrm{~m}, 1 \mathrm{H}), 7.14-7.07(\mathrm{~m}$, $2 \mathrm{H}), 6.61(\mathrm{~s}, 1 \mathrm{H}), 6.12(\mathrm{~s}, 1 \mathrm{H}), 5.84(\mathrm{ddd}, J=10.2,5.0,1.7 \mathrm{~Hz}, 1 \mathrm{H}), 5.483(\mathrm{~s}, 1 \mathrm{H}), 5.477(\mathrm{~s}, 3 \mathrm{H}), 5.28(\mathrm{~d}, J=10.2 \mathrm{~Hz}, 1 \mathrm{H}), 4.11$ $-3.96(\mathrm{~m}, 2 \mathrm{H}), 3.81(\mathrm{~s}, 3 \mathrm{H}), 3.79(\mathrm{~s}, 3 \mathrm{H}), 3.72(\mathrm{~s}, 1 \mathrm{H}), 3.54(\mathrm{~d}, \mathrm{~J}=16.5 \mathrm{~Hz}, 1 \mathrm{H}), 3.45-3.15(\mathrm{~m}, 6 \mathrm{H}), 3.12-2.93(\mathrm{~m}, 2 \mathrm{H}), 2.87$ $-2.73(\mathrm{~m}, 3 \mathrm{H}), 2.71(\mathrm{~s}, 3 \mathrm{H}), 2.64(\mathrm{~s}, 1 \mathrm{H}), 2.59(\mathrm{~d}, J=13.0 \mathrm{~Hz}, 1 \mathrm{H}), 2.49-2.39(\mathrm{~m}, 2 \mathrm{H}), 2.10(\mathrm{~s}, 3 \mathrm{H}), 1.93(\mathrm{q}, J=7.9 \mathrm{~Hz}, 2 \mathrm{H})$, $1.87-1.75(\mathrm{~m}, 2 \mathrm{H}), 1.31(\mathrm{dt}, J=14.1,7.1 \mathrm{~Hz}, 1 \mathrm{H}), 1.13(\mathrm{t}, J=7.1 \mathrm{~Hz}, 3 \mathrm{H}), 0.99(\mathrm{t}, J=7.5 \mathrm{~Hz}, 3 \mathrm{H}), 0.80(\mathrm{t}, J=7.4 \mathrm{~Hz}, 3 \mathrm{H})$. LRMS (ESI): calcd [M+H] $]^{+}\left(\mathrm{C}_{47} \mathrm{H}_{58} \mathrm{~N}_{4} \mathrm{O}_{8}\right)$ 807.4, found 807.4.

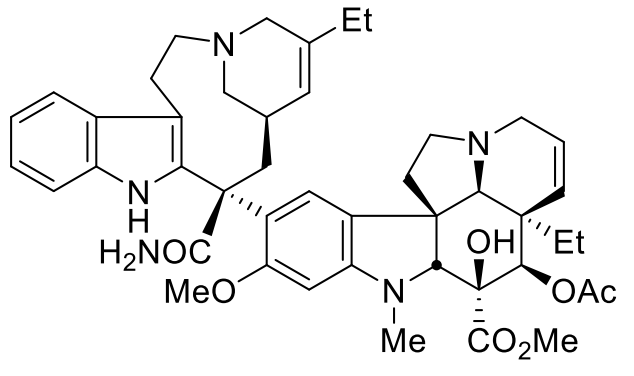


Compound 14b. Following general procedure A, 11b $(6.4 \mathrm{mg}, 0.02 \mathrm{mmol})$, vindoline $(3,27.4 \mathrm{mg}, 0.06 \mathrm{mmol})$, and BAHA $(6,18.0 \mathrm{mg}, 0.022 \mathrm{mmol})$ were combined and the reaction conducted in HFIP $(0.38 \mathrm{~mL})$ and aq $0.05 \mathrm{~N} \mathrm{HCl}(0.76 \mathrm{~mL})$. PTLC $\left(\mathrm{SiO}_{2}, 15 \% \mathrm{MeOH} / \mathrm{EtOAC}\right)$ gave $9.0 \mathrm{mg}\left(58 \%\right.$ yield) of $14 \mathrm{~b}$ identical in all respects with reported material: ${ }^{4} \mathrm{H} \mathrm{NMR}(600$ $\left.\mathrm{MHz}, \mathrm{CDCl}_{3}\right) \delta 9.83(\mathrm{bs}, 1 \mathrm{H}), 8.36(\mathrm{~s}, 1 \mathrm{H}), 7.53(\mathrm{~d}, J=7.9 \mathrm{~Hz}, 1 \mathrm{H}), 7.21-7.07(\mathrm{~m}, 3 \mathrm{H}), 6.74(\mathrm{~s}, 1 \mathrm{H}), 6.15(\mathrm{~s}, 1 \mathrm{H}), 5.83(\mathrm{dd}, J=$ 10.7, $4.9 \mathrm{~Hz}, 1 \mathrm{H}), 5.65-5.15$ (bs, 2H) $5.47-5.45(\mathrm{~m}, 2 \mathrm{H}), 5.25(\mathrm{~d}, J=10.1 \mathrm{~Hz}, 1 \mathrm{H}), 3.85-3.80(\mathrm{~m}, 3 \mathrm{H}), 3.79(\mathrm{~s}, 3 \mathrm{H}), 3.74$ $(\mathrm{s}, 1 \mathrm{H}), 3.45(\mathrm{~d}, J=16.6 \mathrm{~Hz}, 1 \mathrm{H}), 3.37(\mathrm{~d}, J=16.9 \mathrm{~Hz}, 1 \mathrm{H}), 3.33-3.03(\mathrm{~m}, 7 \mathrm{H}), 2.92-2.79(\mathrm{~m}, 2 \mathrm{H}), 2.71(\mathrm{~s}, 3 \mathrm{H}), 2.66-2.62$ $(\mathrm{m}, 2 \mathrm{H}), 2.51-2.38(\mathrm{~m}, 2 \mathrm{H}), 2.25-2.14(\mathrm{~m}, 1 \mathrm{H}), 2.09(\mathrm{~s}, 3 \mathrm{H}), 2.00-1.86(\mathrm{~m}, 1 \mathrm{H}), 1.91(\mathrm{q}, J=7.5 \mathrm{~Hz}, 2 \mathrm{H}), 1.75(\mathrm{dq}, J=14.9$, $7.7 \mathrm{~Hz}, 1 \mathrm{H}), 1.62-1.53(\mathrm{~m}, 1 \mathrm{H}), 1.48-1.43(\mathrm{~m}, 1 \mathrm{H}), 0.98(\mathrm{t}, J=7.5 \mathrm{~Hz}, 3 \mathrm{H}), 0.69(\mathrm{t}, J=7.0 \mathrm{~Hz}, 3 \mathrm{H})$; LRMS (ESI): calcd [M+H] $\left(\mathrm{C}_{45} \mathrm{H}_{56} \mathrm{~N}_{5} \mathrm{O}_{7}\right) 778.4$, found 778.4 .

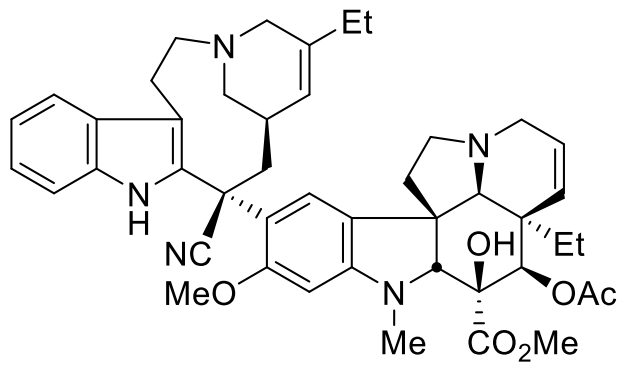

Compound 14c. Following general procedure A, 11c $(6.1 \mathrm{mg}, 0.02 \mathrm{mmol})$, vindoline $(3,9.1 \mathrm{mg}, 0.02 \mathrm{mmol})$, and BAHA (6, $18.0 \mathrm{mg}, 0.022 \mathrm{mmol}$ ) were combined and the reaction conducted in HFIP $(0.38 \mathrm{~mL})$ and aq $0.05 \mathrm{~N} \mathrm{HCl}(0.76 \mathrm{~mL})$. PTLC $\left(\mathrm{SiO}_{2}, 5 \% \mathrm{MeOH} / \mathrm{EtOAc}\right)$ gave $11.5 \mathrm{mg}$ (76\% yield) of $14 \mathrm{c}$ as a white solid identical in all respects with reported material: ${ }^{4}$ ${ }^{1} \mathrm{H}$ NMR $\left(600 \mathrm{MHz}, \mathrm{CDCl}_{3}\right) \delta 9.57(\mathrm{~s}, 1 \mathrm{H}), 7.54-7.50(\mathrm{~m}, 1 \mathrm{H}), 7.21(\mathrm{~s}, 1 \mathrm{H}), 7.10-6.94(\mathrm{~m}, 4 \mathrm{H}), 6.05(\mathrm{~s}, 1 \mathrm{H}), 5.91(\mathrm{dd}, J=$ $10.2,4.3 \mathrm{~Hz}, 1 \mathrm{H}), 5.45(\mathrm{~s}, 1 \mathrm{H}), 5.41(\mathrm{~d}, J=5.4 \mathrm{~Hz}, 1 \mathrm{H}), 5.26(\mathrm{dt}, J=10.0,1.9 \mathrm{~Hz}, 1 \mathrm{H}), 3.79(\mathrm{~s}, 3 \mathrm{H}), 3.79-3.74(\mathrm{~m}, 1 \mathrm{H}), 3.64$ $-3.53(\mathrm{~m}, 2 \mathrm{H}), 3.59(\mathrm{~s}, 3 \mathrm{H}), 3.52-3.46(\mathrm{~m}, 1 \mathrm{H}), 3.34(\mathrm{~d}, J=15.6 \mathrm{~Hz}, 1 \mathrm{H}), 3.11(\mathrm{~d}, J=15.7 \mathrm{~Hz}, 1 \mathrm{H}), 2.93-2.88(\mathrm{~m}, 2 \mathrm{H}), 2.79$ $(\mathrm{d}, J=13.4 \mathrm{~Hz}, 1 \mathrm{H}), 2.75(\mathrm{~s}, 1 \mathrm{H}), 2.72-2.58(\mathrm{~m}, 3 \mathrm{H}), 2.66(\mathrm{~s}, 3 \mathrm{H}), 2.51-2.34(\mathrm{~m}, 5 \mathrm{H}), 2.22(\mathrm{~d}, J=11.2 \mathrm{~Hz}, 1 \mathrm{H}), 2.09(\mathrm{~s}, 3 \mathrm{H})$, $2.01(\mathrm{q}, J=7.8 \mathrm{~Hz}, 2 \mathrm{H}), 1.85(\mathrm{dq}, J=14.1,6.8 \mathrm{~Hz}, 1 \mathrm{H}), 1.23-1.16(\mathrm{~m}, 1 \mathrm{H}), 1.03(\mathrm{t}, J=7.5 \mathrm{~Hz}, 3 \mathrm{H}), 0.75(\mathrm{t}, J=7.4 \mathrm{~Hz}, 3 \mathrm{H})$; ${ }^{13} \mathrm{C} \mathrm{NMR}\left(150 \mathrm{MHz}, \mathrm{CDCl}_{3}\right) \delta 171.9,171.0,159.6,153.9,142.2,134.7,133.0,130.2,128.3,124.8,124.0,122.4,121.9,121.5$, $119.8,119.3,118.5,111.8,110.2,96.0,83.5,79.5,76.4,67.1,56.3,55.4,53.2,52.7,52.5,52.2,51.8,51.2,45.9,44.0,43.1$, 41.4, 38.3, 35.1, 31.1, 29.8, 27.5, 25.2, 21.2, 12.3, 8.7; HRMS (ESI-TOF): calcd $[\mathrm{M}+\mathrm{H}]^{+}\left(\mathrm{C}_{45} \mathrm{H}_{54} \mathrm{~N}_{5} \mathrm{O}_{6}\right)$ 760.4074, found 760.4082 .

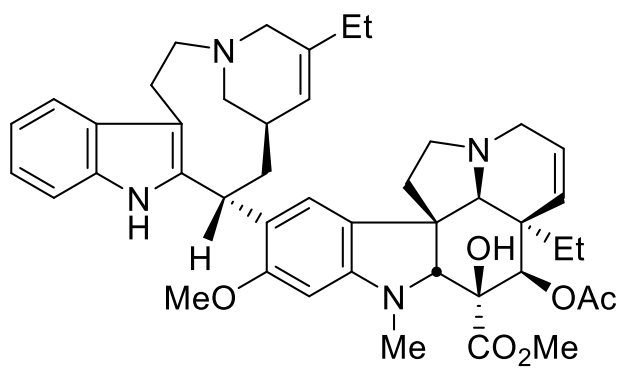

Compound 14d. Following general procedure A, 11d (5.6 mg, $0.02 \mathrm{mmol})$, vindoline (3, $9.1 \mathrm{mg}, 0.02 \mathrm{mmol})$, and BAHA (6, $18.0 \mathrm{mg}, 0.022 \mathrm{mmol}$ ) were combined and the reaction conducted in $\mathrm{HFIP}(0.38 \mathrm{~mL})$ and aq $0.05 \mathrm{~N} \mathrm{HCl}(0.76 \mathrm{~mL})$. PTLC $\left(\mathrm{SiO}_{2}, 5 \% \mathrm{MeOH} / \mathrm{EtOAc}\right)$ gave $1.8 \mathrm{mg}\left(12 \%\right.$ yield) of $14 \mathrm{~d}$ as a white solid: ${ }^{1} \mathrm{H} \mathrm{NMR}\left(600 \mathrm{MHz}, \mathrm{CDCl}_{3}\right) \delta 9.59(\mathrm{bs}, 1 \mathrm{H}), 8.65(\mathrm{~s}$, $1 \mathrm{H}), 7.45(\mathrm{~d}, J=7.3 \mathrm{~Hz}, 1 \mathrm{H}), 7.16(\mathrm{~d}, J=7.4 \mathrm{~Hz}, 1 \mathrm{H}), 7.08-6.97(\mathrm{~m}, 2 \mathrm{H}), 6.82(\mathrm{~s}, 1 \mathrm{H}), 6.10(\mathrm{~s}, 1 \mathrm{H}), 5.88(\mathrm{ddd}, J=10.1,4.9$, $1.7 \mathrm{~Hz}, 1 \mathrm{H}), 5.56(\mathrm{~d}, J=9.9 \mathrm{~Hz}, 1 \mathrm{H}), 5.44(\mathrm{~s}, 1 \mathrm{H}), 5.23(\mathrm{~d}, J=10.1 \mathrm{~Hz}, 1 \mathrm{H}), 5.20(\mathrm{bd}, J=4.1 \mathrm{~Hz}, 1 \mathrm{H}), 3.91(\mathrm{~s}, 3 \mathrm{H}), 3.78(\mathrm{~s}, 3 \mathrm{H})$, $3.66(\mathrm{~s}, 1 \mathrm{H}), 3.52(\mathrm{ddd}, J=15.8,4.7,1.0 \mathrm{~Hz}, 1 \mathrm{H}), 3.43(\mathrm{td}, J=9.1,4.0 \mathrm{~Hz}, 1 \mathrm{H}), 3.24(\mathrm{~d}, J=15.3 \mathrm{~Hz}, 1 \mathrm{H}), 3.12(\mathrm{~d}, J=15.3 \mathrm{~Hz}$, $1 \mathrm{H}), 2.99(\mathrm{td}, J=12.8,3.7 \mathrm{~Hz}, 1 \mathrm{H}), 2.90-2.85(\mathrm{~m}, 2 \mathrm{H}), 2.78(\mathrm{~d}, J=13.7 \mathrm{~Hz}, 1 \mathrm{H}), 2.75-2.67(\mathrm{~m}, 2 \mathrm{H}), 2.62(\mathrm{~s}, 3 \mathrm{H}), 2.57(\mathrm{q}, J$ $=9.7 \mathrm{~Hz}, 1 \mathrm{H}), 2.51-2.41(\mathrm{~m}, 3 \mathrm{H}), 2.36-2.24(\mathrm{~m}, 2 \mathrm{H}), 2.20-2.15(\mathrm{~m}, 1 \mathrm{H}), 2.08(\mathrm{~s}, 3 \mathrm{H}), 2.03-1.95(\mathrm{~m}, 3 \mathrm{H}), 1.72-1.54(\mathrm{~m}$, $2 \mathrm{H}), 1.02(\mathrm{t}, J=7.5 \mathrm{~Hz}, 3 \mathrm{H}), 0.56(\mathrm{t}, J=7.3 \mathrm{~Hz}, 3 \mathrm{H}) ;{ }^{13} \mathrm{C} \mathrm{NMR}\left(150 \mathrm{MHz}, \mathrm{CDCl}_{3}\right) \delta$ 172.1, 171.0, 158.3, 152.1, 142.2, 141.0, 135.4, 130.6, 128.4, 127.2, 124.6, 124.4, 122.8, 122.5, 120.6, 118.5, 117.9, 110.2, 109.3, 95.3, 83.8, 79.7, 76.6, 67.1, 56.3, $55.1,53.9,53.2,52.4,52.2,51.3,44.0,43.1,40.7,39.2,35.6,35.4,30.9,29.8,27.9,26.9,21.2,12.9,8.1$; IR (neat, ATR) 
2958, 2924, 2850, 1741, 1499, 1461, 1244, $1040 \mathrm{~cm}^{-1}$; HRMS (ESI-TOF): calcd [M+H] $]^{+}\left(\mathrm{C}_{44} \mathrm{H}_{55} \mathrm{~N}_{4} \mathrm{O}_{6}\right)$ 735.4116, found $735.4118 ;[\alpha]_{D}^{24}-6\left(c 0.18, \mathrm{CHCl}_{3}\right)$.

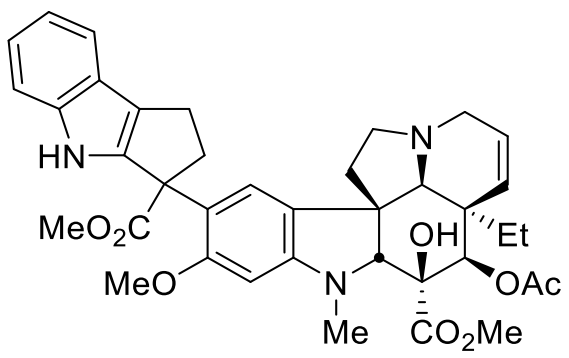

Compound 15. Following general procedure A, $12(4.3 \mathrm{mg}, 0.02 \mathrm{mmol})$, vindoline $(3,9.1 \mathrm{mg}, 0.02 \mathrm{mmol})$, and BAHA (6, $18.0 \mathrm{mg}, 0.022 \mathrm{mmol}$ ) were combined and the reaction conducted in TFE $(0.38 \mathrm{~mL})$ and aq $0.05 \mathrm{~N} \mathrm{HCl}(0.76 \mathrm{~mL})$. PTLC $\left(\mathrm{SiO}_{2}, 80 \% \mathrm{EtOAc} /\right.$ hexanes) gave $6.7 \mathrm{mg}$ (50\% yield) of 15 as a white solid as a 1:1.5 diastereomeric mixture $\left(3^{\prime} R-15: 3^{\prime} S-\right.$ 15) identical in all respects with reported material. ${ }^{9}$ For 3 'S-15 (less polar diastereomer): ${ }^{1} \mathrm{H} \mathrm{NMR}\left(600 \mathrm{MHz}, \mathrm{CDCl}_{3}\right) \delta 9.69$ (bs, $1 \mathrm{H}$ ), $7.81(\mathrm{~s}, 1 \mathrm{H}), 7.52$ (d, $J=7.7 \mathrm{~Hz}, 1 \mathrm{H}), 7.30$ (d, $J=8.1 \mathrm{~Hz}, 1 \mathrm{H}$ ), 7.18 (ddd, $J=8.2,7.1,1.3 \mathrm{~Hz}, 1 \mathrm{H}$ ), 7.13 (ddd, $J=8.2$, 7.1, $1.1 \mathrm{~Hz}, 1 \mathrm{H}), 6.55(\mathrm{~s}, 1 \mathrm{H}), 6.12(\mathrm{~s}, 1 \mathrm{H}), 5.88$ (ddd, J = 10.2, 5.0, $1.6 \mathrm{~Hz}, 1 \mathrm{H}), 5.38(\mathrm{~s}, 1 \mathrm{H}), 5.29(\mathrm{~d}, J=10.2 \mathrm{~Hz}, 1 \mathrm{H}), 3.86(\mathrm{~s}$, $3 \mathrm{H}), 3.80(\mathrm{~s}, 3 \mathrm{H}), 3.75(\mathrm{~s}, 1 \mathrm{H}), 3.69(\mathrm{~s}, 3 \mathrm{H}), 3.63$ (ddd, $J=13.4,8.6,5.1 \mathrm{~Hz}, 1 \mathrm{H}), 3.42(\mathrm{dd}, J=15.5,4.2 \mathrm{~Hz}, 1 \mathrm{H}), 3.39-3.34$ ( $\mathrm{m}, 1 \mathrm{H}$ ), 3.00 (ddd, $J=14.2,8.6,4.7 \mathrm{~Hz}, 1 \mathrm{H}), 2.86$ (ddd, $J=14.0,8.4,5.1 \mathrm{~Hz}, 1 \mathrm{H}), 2.81(\mathrm{bd}, J=15.9 \mathrm{~Hz}, 1 \mathrm{H}), 2.74(\mathrm{~s}, 3 \mathrm{H})$, 2.66 (bs, 1H), $2.55-2.45(\mathrm{~m}, 2 \mathrm{H}), 2.23-2.13(\mathrm{~m}, 1 \mathrm{H}), 2.10(\mathrm{~s}, 3 \mathrm{H}), 2.06-2.01(\mathrm{~m}, 1 \mathrm{H}), 1.70-1.61(\mathrm{~m}, 3 \mathrm{H}), 1.20(\mathrm{dq}, J=$ $14.3,7.2 \mathrm{~Hz}, 1 \mathrm{H}), 0.59(\mathrm{t}, J=7.4 \mathrm{~Hz}, 3 \mathrm{H})$; LRMS (ESI): calcd $[\mathrm{M}+\mathrm{H}]^{+}\left(\mathrm{C}_{38} \mathrm{H}_{44} \mathrm{~N}_{3} \mathrm{O}_{8}\right.$ ) 670.3, found 670.3. For 3'R-15 (more polar diastereomer): ${ }^{1} \mathrm{H} \mathrm{NMR}\left(600 \mathrm{MHz}, \mathrm{CDCl}_{3}\right) \delta 9.71(\mathrm{bs}, 1 \mathrm{~Hz}), 8.02(\mathrm{~s}, 1 \mathrm{H}), 7.50(\mathrm{dd}, J=7.8,1.1 \mathrm{~Hz}, 1 \mathrm{H}), 7.35(\mathrm{~d}, J=8.2 \mathrm{~Hz}, 1 \mathrm{H})$, 7.17 (ddd, $J=8.2,7.1,1.3 \mathrm{~Hz}, 1 \mathrm{H}$ ), 7.11 (ddd, $J=8.1,7.1,1.1 \mathrm{~Hz}, 1 \mathrm{H}$ ), $6.51(\mathrm{~s}, 1 \mathrm{H}), 6.11(\mathrm{~s}, 1 \mathrm{H}$ ), 5.78 (ddd, $J=10.2,4.8,1.7$ $\mathrm{Hz}, 1 \mathrm{H}), 5.39(\mathrm{~s}, 1 \mathrm{H}), 5.15(\mathrm{~d}, J=9.9 \mathrm{~Hz}, 1 \mathrm{H}), 3.83(\mathrm{~s}, 3 \mathrm{H}), 3.78(\mathrm{~s}, 3 \mathrm{H}), 3.73(\mathrm{~s}, 1 \mathrm{H}), 3.67(\mathrm{~s}, 3 \mathrm{H}), 3.58(\mathrm{ddd}, J=13.4,8.5,5.2$ $\mathrm{Hz}, 1 \mathrm{H}), 3.39(\mathrm{dd}, J=16.2,4.7 \mathrm{~Hz}, 1 \mathrm{H}), 3.35-3.30(\mathrm{~m}, 3 \mathrm{H}), 2.94(\mathrm{ddd}, J=14.2,8.5,4.6 \mathrm{~Hz}, 1 \mathrm{H}), 2.83-2.73(\mathrm{~m}, 2 \mathrm{H}), 2.70$ (s, 3H), $2.48-2.41(\mathrm{~m}, 1 \mathrm{H}), 2.47(\mathrm{~s}, 1 \mathrm{H}), 2.37$ (ddd, $J=13.0,8.3,4.6 \mathrm{~Hz}, 1 \mathrm{H}), 2.27(\mathrm{dt}, J=15.6,8.2 \mathrm{~Hz}, 1 \mathrm{H}), 2.17$ (ddd, $J=$ 14.2, 10.8, 4.2 Hz, 1H), $2.06(\mathrm{~s}, 3 \mathrm{H}), 1.64-1.53(\mathrm{~m}, 1 \mathrm{H}), 0.97(\mathrm{dq}, J=14.5,7.2 \mathrm{~Hz}, 1 \mathrm{H}), 0.33(\mathrm{t}, J=7.4 \mathrm{~Hz}, 3 \mathrm{H})$; LRMS (ESI): calcd $[\mathrm{M}+\mathrm{H}]^{+}\left(\mathrm{C}_{38} \mathrm{H}_{44} \mathrm{~N}_{3} \mathrm{O}_{8}\right) 670.3$, found 670.3 . The structure and stereochemistry of 3'S-15 were established by $\mathrm{X}$-ray crystallography (CCDC 1947271) conducted with colorless crystals obtained from slow vapor diffusion with $\mathrm{MeOH} /$ pentanes. For further X-ray crystallography data refer to Table S1.

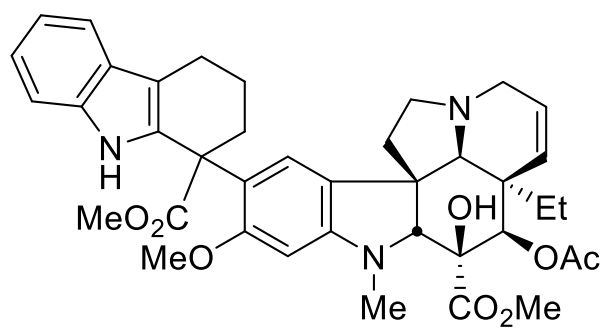

Compound 16. Following general procedure A, $13(9.2 \mathrm{mg}, 0.04 \mathrm{mmol})$, vindoline $(3,9.1 \mathrm{mg}, 0.02 \mathrm{mmol})$, and BAHA (6, $18.0 \mathrm{mg}, 0.022 \mathrm{mmol}$ ) were combined and the reaction conducted in TFE $(0.76 \mathrm{~mL})$ and aq $0.05 \mathrm{~N} \mathrm{HCl}(0.76 \mathrm{~mL})$. PTLC $\left(\mathrm{SiO}_{2}, 3 \% \mathrm{MeOH} / \mathrm{EtOAC}\right.$ ) gave $7.6 \mathrm{mg}$ (55\% yield) of 16 as a 1:1.2 diastereomeric mixture (3'R-16:3'S-16) identical in all respects with reported material. ${ }^{9}$ For 3 'S-16 (less polar diastereomer): ${ }^{1} \mathrm{H}$ NMR $\left(600 \mathrm{MHz}, \mathrm{CDCl}_{3}\right) \delta 9.74(\mathrm{bs}, 1 \mathrm{H}), 7.99(\mathrm{~s}$, $1 \mathrm{H}), 7.54(\mathrm{~d}, J=7.8 \mathrm{~Hz}, 1 \mathrm{H}), 7.18(\mathrm{~d}, J=8.0 \mathrm{~Hz}, 1 \mathrm{H}), 7.15$ (ddd, $J=8.1,6.9,1.3 \mathrm{~Hz}, 1 \mathrm{H}), 7.10(\mathrm{ddd}, J=8.0,6.9,1.2 \mathrm{~Hz}, 1 \mathrm{H})$, $6.13(\mathrm{~s}, 1 \mathrm{H}), 6.09(\mathrm{~s}, 1 \mathrm{H}), 5.80$ (ddd, $J=10.1,5.0,1.6 \mathrm{~Hz}, 1 \mathrm{H}), 5.35(\mathrm{~s}, 1 \mathrm{H}), 5.24(\mathrm{dt}, J=9.9,2.0 \mathrm{~Hz}, 1 \mathrm{H}), 3.83(\mathrm{~s}, 3 \mathrm{H}), 3.77(\mathrm{~s}$, $3 \mathrm{H}), 3.74(\mathrm{~s}, 3 \mathrm{H}), 3.70(\mathrm{~s}, 1 \mathrm{H}), 3.36-3.24(\mathrm{~m}, 2 \mathrm{H}), 2.82-2.67(\mathrm{~m}, 4 \mathrm{H}), 2.71(\mathrm{~s}, 3 \mathrm{H}), 2.50(\mathrm{~s}, 1 \mathrm{H}), 2.40$ (ddd, J = 11.4, $9.8,5.9$ $\mathrm{Hz}, 1 \mathrm{H}), 2.15-2.02(\mathrm{~m}, 2 \mathrm{H}), 2.07(\mathrm{~s}, 3 \mathrm{H}), 1.98-1.85(\mathrm{~m}, 2 \mathrm{H}), 1.68-1.52(\mathrm{~m}, 2 \mathrm{H}), 1.18(\mathrm{dq}, J=14.4,7.2 \mathrm{~Hz}, 1 \mathrm{H}), 0.52(\mathrm{t}, J$ $=7.4 \mathrm{~Hz}, 3 \mathrm{H})$; LRMS (ESI): calcd $[\mathrm{M}+\mathrm{H}]^{+}\left(\mathrm{C}_{39} \mathrm{H}_{46} \mathrm{~N}_{3} \mathrm{O}_{8}\right)$ 684.3, found 684.3. For $3^{\prime} R-16$ (more polar diastereomer): ${ }^{1} \mathrm{H} \mathrm{NMR}$ $\left(600 \mathrm{MHz}, \mathrm{CDCl}_{3}\right) \delta 9.76(\mathrm{bs}, 1 \mathrm{H}), 8.22(\mathrm{~s}, 1 \mathrm{H}), 7.54(\mathrm{~d}, J=7.5 \mathrm{~Hz}, 1 \mathrm{H}), 7.29(\mathrm{~d}, J=8.1 \mathrm{~Hz}, 1 \mathrm{H}), 7.18$ (ddd, J = 8.2, 7.0, 1.2 Hz, $1 \mathrm{H}), 7.11(\mathrm{t}, J=7.5 \mathrm{~Hz}, 1 \mathrm{H}), 6.12(\mathrm{~s}, 1 \mathrm{H}), 6.09(\mathrm{~s}, 1 \mathrm{H}), 5.81(\mathrm{ddd}, J=10.2,5.0,1.6 \mathrm{~Hz}, 1 \mathrm{H}), 5.32(\mathrm{~s}, 1 \mathrm{H}), 5.23(\mathrm{~d}, J=10.1 \mathrm{~Hz}$, 
$1 \mathrm{H}), 3.83(\mathrm{~s}, 3 \mathrm{H}), 3.77(\mathrm{~s}, 3 \mathrm{H}), 3.75(\mathrm{~s}, 3 \mathrm{H}), 3.70(\mathrm{~s}, 1 \mathrm{H}), 3.35-3.30(\mathrm{~m}, 1 \mathrm{H}), 3.30-3.24(\mathrm{~m}, 1 \mathrm{H}), 2.82(\mathrm{bd}, J=15.9 \mathrm{~Hz}, 1 \mathrm{H})$, $2.72(\mathrm{~s}, 3 \mathrm{H}), 2.72-2.69(\mathrm{~m}, 2 \mathrm{H}), 2.64(\mathrm{ddd}, J=13.4,12.1,2.6 \mathrm{~Hz}, 1 \mathrm{H}), 2.53(\mathrm{~s}, 1 \mathrm{H}), 2.41(\mathrm{td}, J=10.6,5.8 \mathrm{~Hz}, 1 \mathrm{H}), 2.20-$ $2.06(\mathrm{~m}, 2 \mathrm{H}), 2.05(\mathrm{~s}, 3 \mathrm{H}), 1.97-1.84(\mathrm{~m}, 2 \mathrm{H}), 1.57-1.43(\mathrm{~m}, 2 \mathrm{H}), 1.06(\mathrm{dq}, J=14.4,7.2 \mathrm{~Hz}, 1 \mathrm{H}), 0.42(\mathrm{t}, J=7.3 \mathrm{~Hz}, 3 \mathrm{H})$; LRMS (ESI): calcd [M+H] ${ }^{+}\left(\mathrm{C}_{39} \mathrm{H}_{46} \mathrm{~N}_{3} \mathrm{O}_{8}\right) 684.3$, found 684.3. The structure and stereochemistry of $3^{\prime} R$-16 were established by X-ray crystallography (CCDC 1947270) conducted with colorless crystals obtained from slow vapor diffusion with benzene/pentanes. For further X-ray crystallography data refer to Table S2.

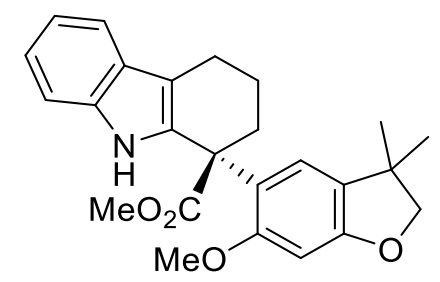

Compound 17. Following general procedure A, $13(4.6 \mathrm{mg}, 0.02 \mathrm{mmol}), \mathbf{S 3}(3.6 \mathrm{mg}, 0.02 \mathrm{mmol})$, and BAHA (6, $18.0 \mathrm{mg}$, $0.022 \mathrm{mmol})$ were combined and the reaction conducted in TFE $(0.76 \mathrm{~mL})$ and aq $0.05 \mathrm{~N} \mathrm{HCl}(0.76 \mathrm{~mL}) . \mathrm{PTLC}_{(\mathrm{SiO}}, 20 \%$ EtOAc/hexanes) gave $4.6 \mathrm{mg}$ (57\% yield) of 17 as a white solid: ${ }^{1} \mathrm{H} \mathrm{NMR}\left(600 \mathrm{MHz}, \mathrm{CDCl}_{3}\right) \delta 8.26(\mathrm{~s}, 1 \mathrm{H}), 7.56(\mathrm{dd}, J=7.8$, $1.0 \mathrm{~Hz}, 1 \mathrm{H}$ ), 7.31 (d, $J=8.1 \mathrm{~Hz}, 1 \mathrm{H}), 7.19$ (ddd, $J=8.1,7.0,1.2 \mathrm{~Hz}, 1 \mathrm{H}), 7.12$ (ddd, J = 8.0, 7.0, $1.0 \mathrm{~Hz}, 1 \mathrm{H}), 6.36(\mathrm{~s}, 1 \mathrm{H}), 6.22$ $(\mathrm{d}, J=1.1 \mathrm{~Hz}, 1 \mathrm{H}), 3.78(\mathrm{~s}, 3 \mathrm{H}), 3.77(\mathrm{~s}, 3 \mathrm{H}), 2.85-2.66(\mathrm{~m}, 5 \mathrm{H}), 2.15(\mathrm{ddd}, J=13.4,5.9,2.5 \mathrm{~Hz}, 1 \mathrm{H}), 1.93-1.85(\mathrm{~m}, 1 \mathrm{H})$, $1.63-1.57(\mathrm{~m}, 1 \mathrm{H}), 1.42(\mathrm{~s}, 3 \mathrm{H}), 1.40(\mathrm{~s}, 3 \mathrm{H}) ;{ }^{13} \mathrm{C} \mathrm{NMR}\left(150 \mathrm{MHz}, \mathrm{CDCl}_{3}\right) \delta 175.4,159.2,156.5,135.9,131.2,127.4,125.9$, 124.5, 122.1, 119.3, 118.7, 117.8, 114.2, 111.1, 94.2, 87.9, 55.7, 52.5, 50.8, 42.6, 32.6, 28.5, 28.4, 21.2, 19.7; IR (neat, ATR) 3437, 2929, 2846, 1731, 1618, 1486, 1458, 1196, 1091, $740 \mathrm{~cm}^{-1}$; HRMS (ESI-TOF): calcd [M+H] ${ }^{+}\left(\mathrm{C}_{25} \mathrm{H}_{28} \mathrm{NO}_{4}\right) 406.2018$, found 406.2018 


\section{References}

1. Lau, N.; Sano, Y.; Ziller, J. W.; Borovik, A. S. Terminal Ni"-OH/-OH $\mathrm{OH}_{2}$ complexes in trigonal bipyramidal geometries derived from $\mathrm{H}_{2} \mathrm{O}$. Polyhedron 2017, 125, 179-185.

2. Barton, D. H. R.; Haynes, R. K.; Leclerc, G.; Magnus, P. D.; Menzies, I. D. New reactions of triplet oxygen which avoid the spin barrier. J. Chem. Soc., Perkin Trans. 1 1975, 2055-2065.

3. lida, K.; Yoshida, J.-i. Radical polymerization initiated by electron transfer driven C-Si bond dissociation. Macromolecules 2006, 39, 6420-6424.

4. Tam, A.; Gotoh, H.; Robertson, W. M.; Boger, D. L. Catharanthine C16 substituent effects on the biomimetic coupling with vindoline: preparation and evaluation of a key series of vinblastine analogues. Bioorg. Med. Chem. Lett. 2010, 20, 6408-6410.

5. Maruyama, J.; Yamashita, H.; Watanabe, T.; Arai, S.; Nishida, A. Novel synthesis of fused indoles and 2-substituted indoles by the palladium-catalyzed cyclization of N-cycloalkenyl-o-haloanilines. Tetrahedron 2009, 65, 1327-1335.

6. Hardouin, C.; Doris, E.; Rousseau, B.; Mioskowski, C. Selective deoxygenation of leurosine: concise access to anhydrovinblastine. J. Org. Chem. 2002, 67, 6571-6574.

7. Ishikawa, H.; Colby, D. A.; Boger, D. L. Direct coupling of catharanthine and vindoline to provide vinblastine: Total synthesis of (+)- and ent-(-)-vinblastine. J. Am. Chem. Soc. 2008, 130, 420-421.

8. Chen, Z.; Jiang, Y.; Zhang, L.; Guo, Y.; Ma, D. Oxalic diamides and tert-butoxide: Two types of ligands enabling practical access to alkyl aryl ethers via Cu-catalyzed coupling reaction. J. Am. Chem. Soc. 2019, 141, 3541-3549.

9. Gotoh, H.; Sears, J. E.; Eschenmoser, A.; Boger, D. L. New insights into the mechanism and an expanded scope of the $\mathrm{Fe}$ (III)-mediated vinblastine coupling reaction. J. Am. Chem. Soc. 2012, 134, 13240-13243.

10. Goossens, R.; Lhomeau, G.; Forier, B.; Toppet, S.; Meervelt, L. V.; Dehaen, W. Acid-catalyzed condensation of citronellal and electron rich phenols: Mechanism and functionalization of the adducts. Tetrahedron 2000, 56, 9297-9303. 

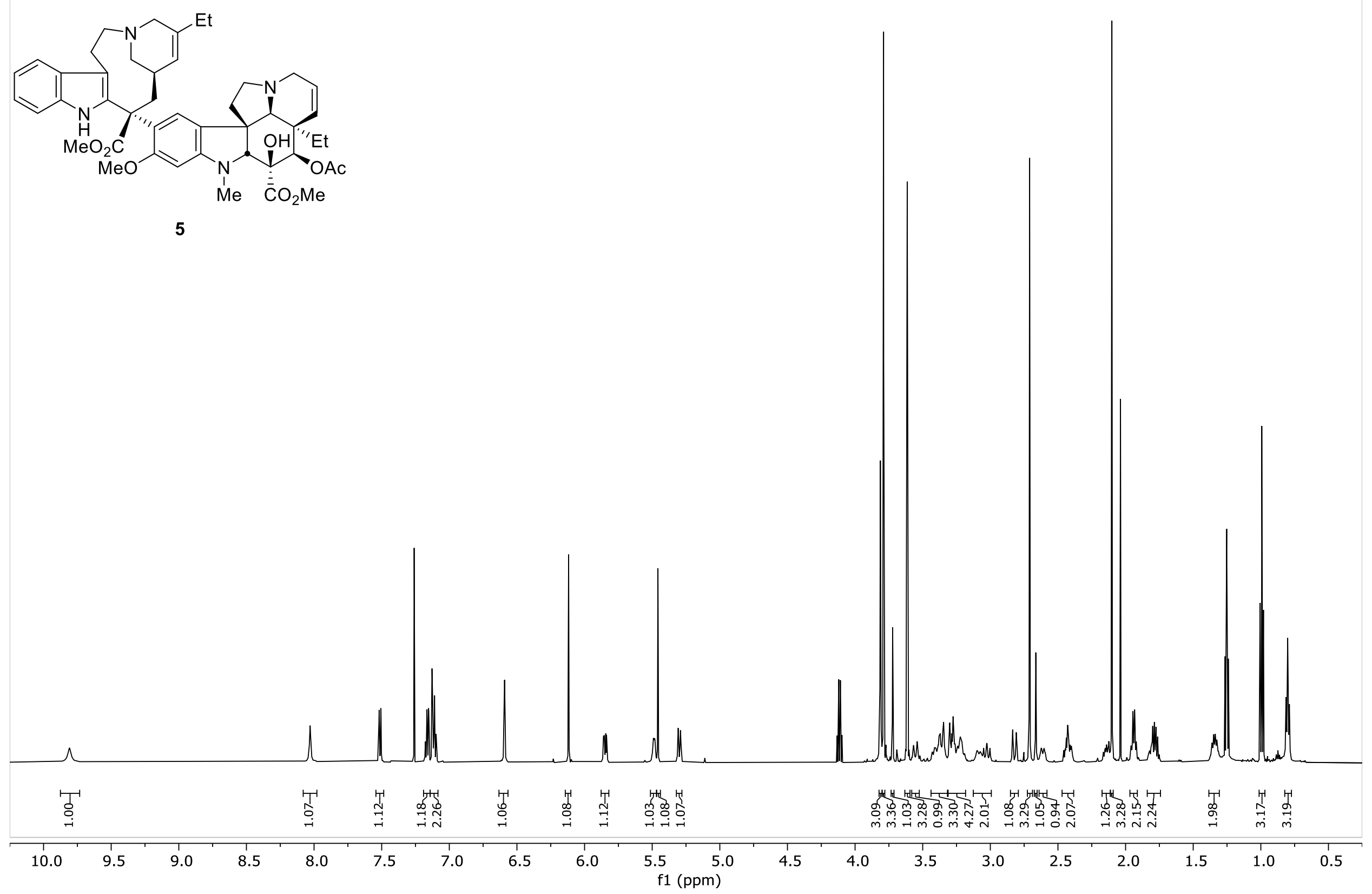

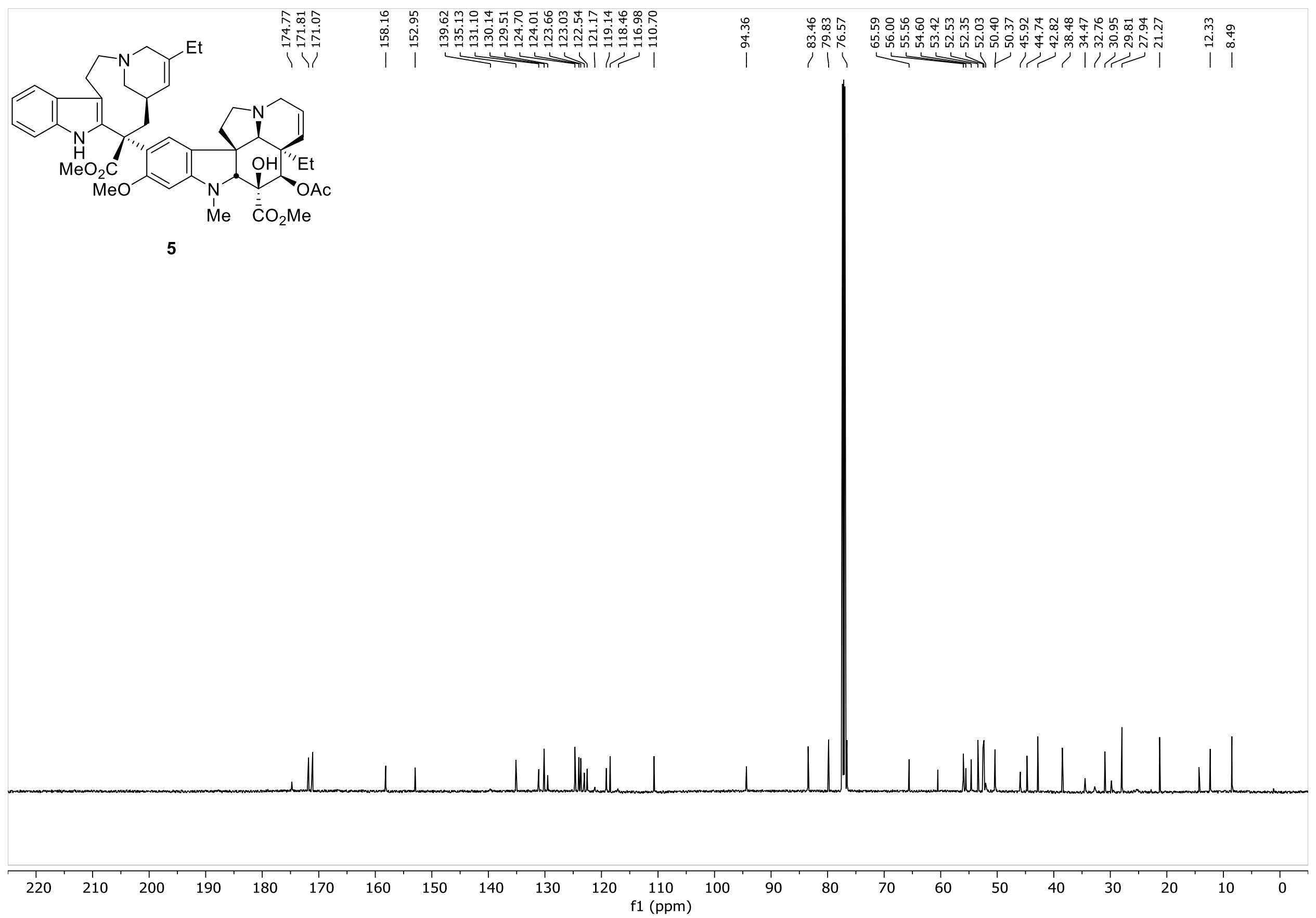


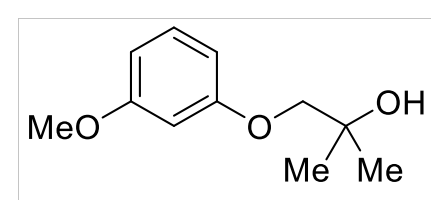

S2

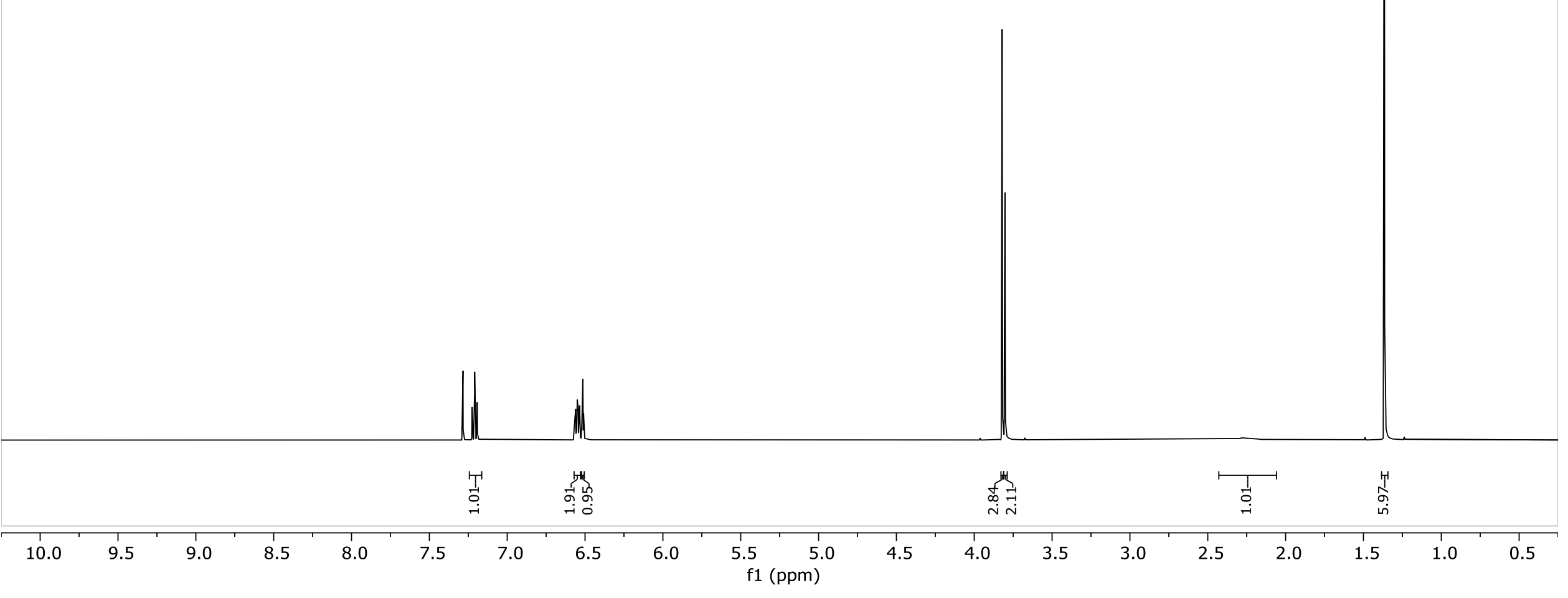




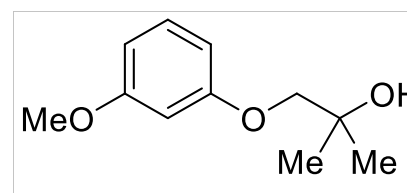

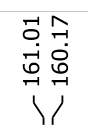

$\stackrel{\infty}{\stackrel{\infty}{0}}$

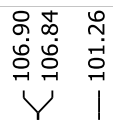

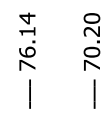

站

$\underset{\substack{0 \\ \text { i }}}{1}$

S2

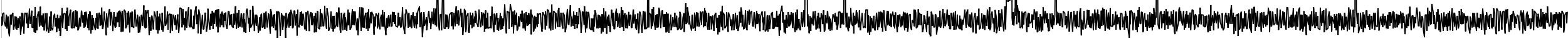

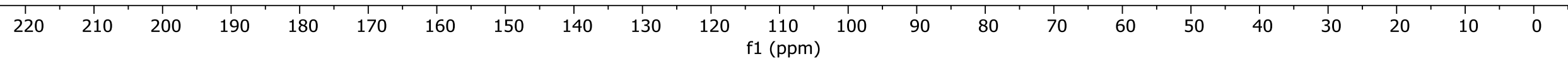




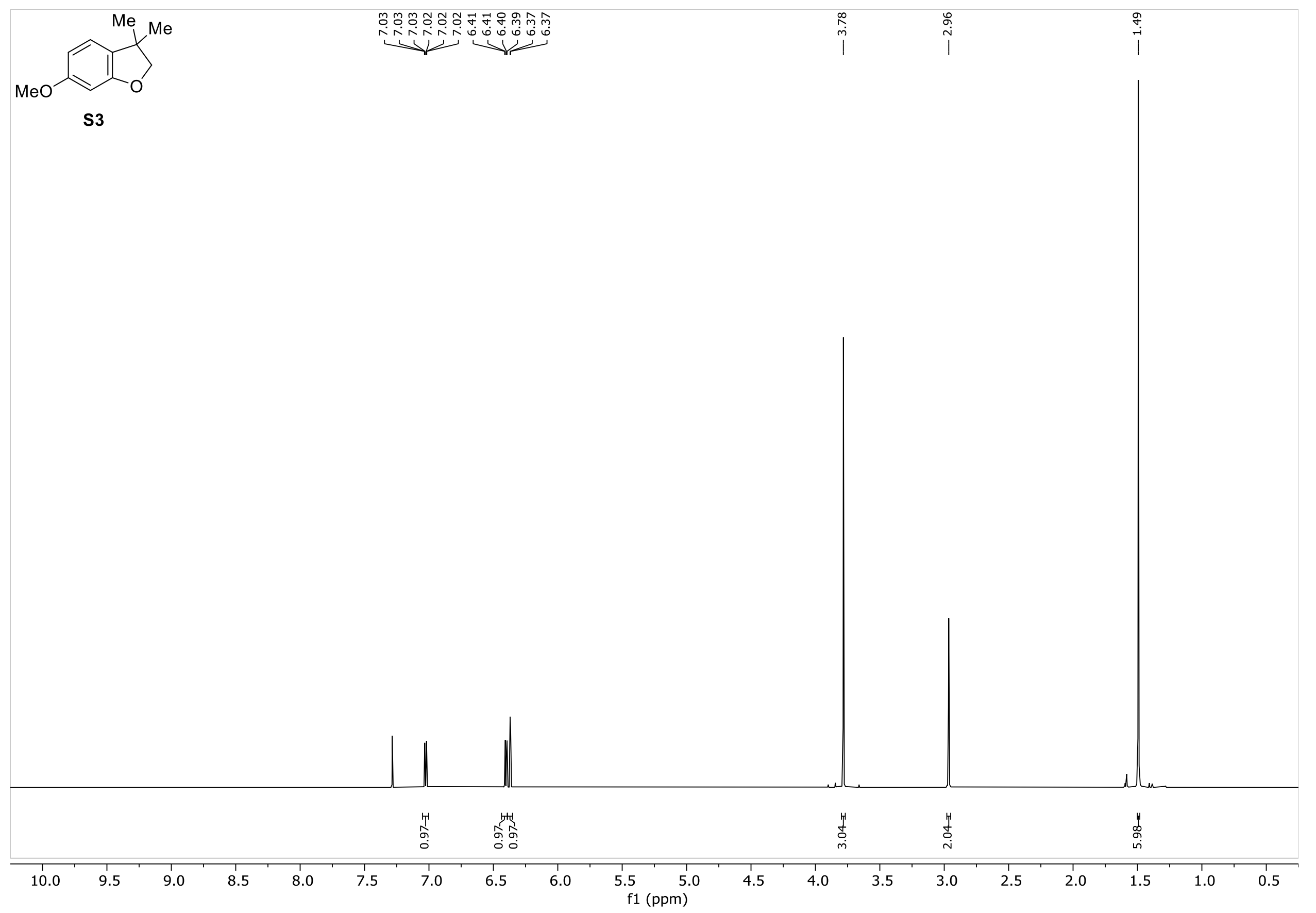



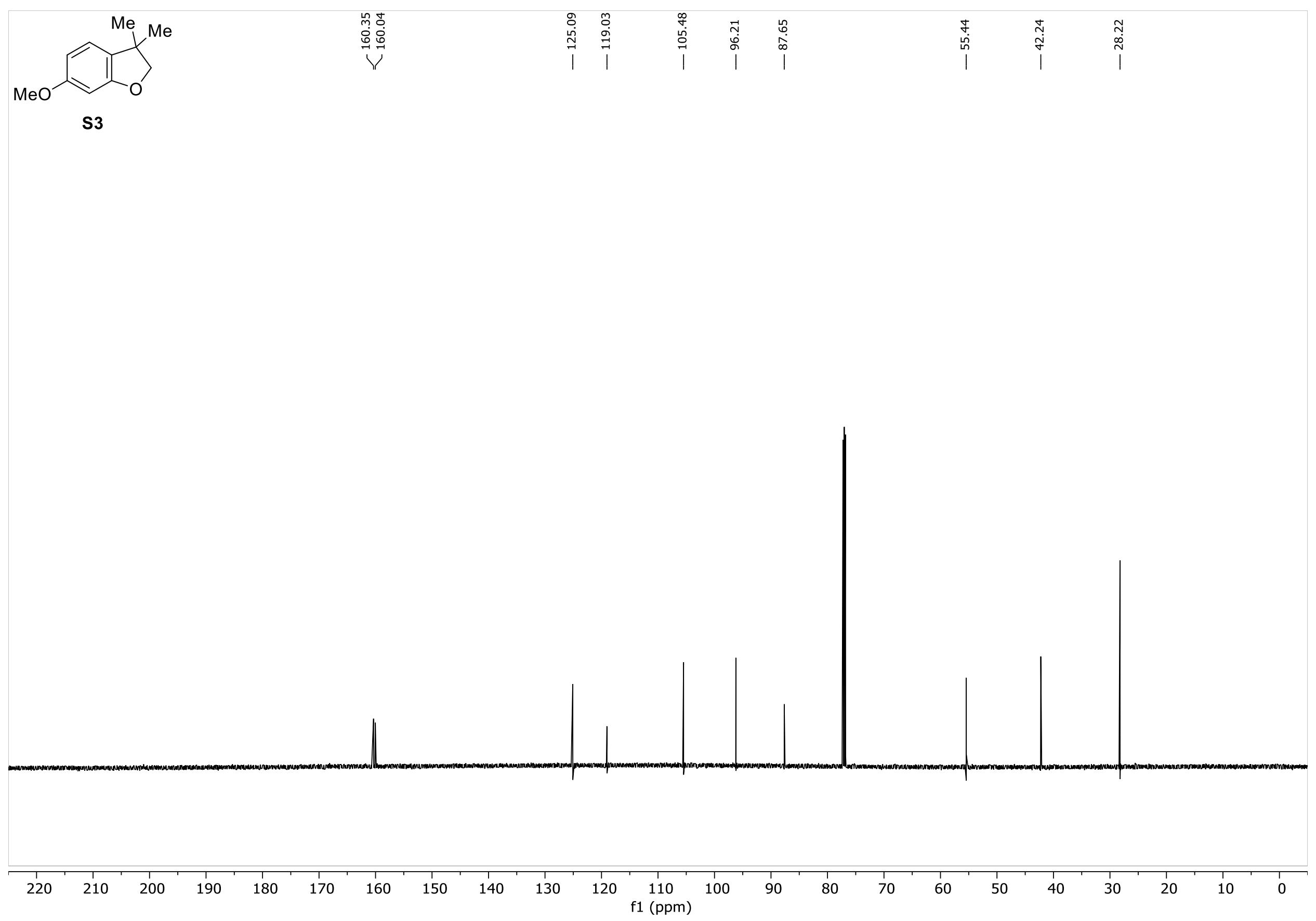


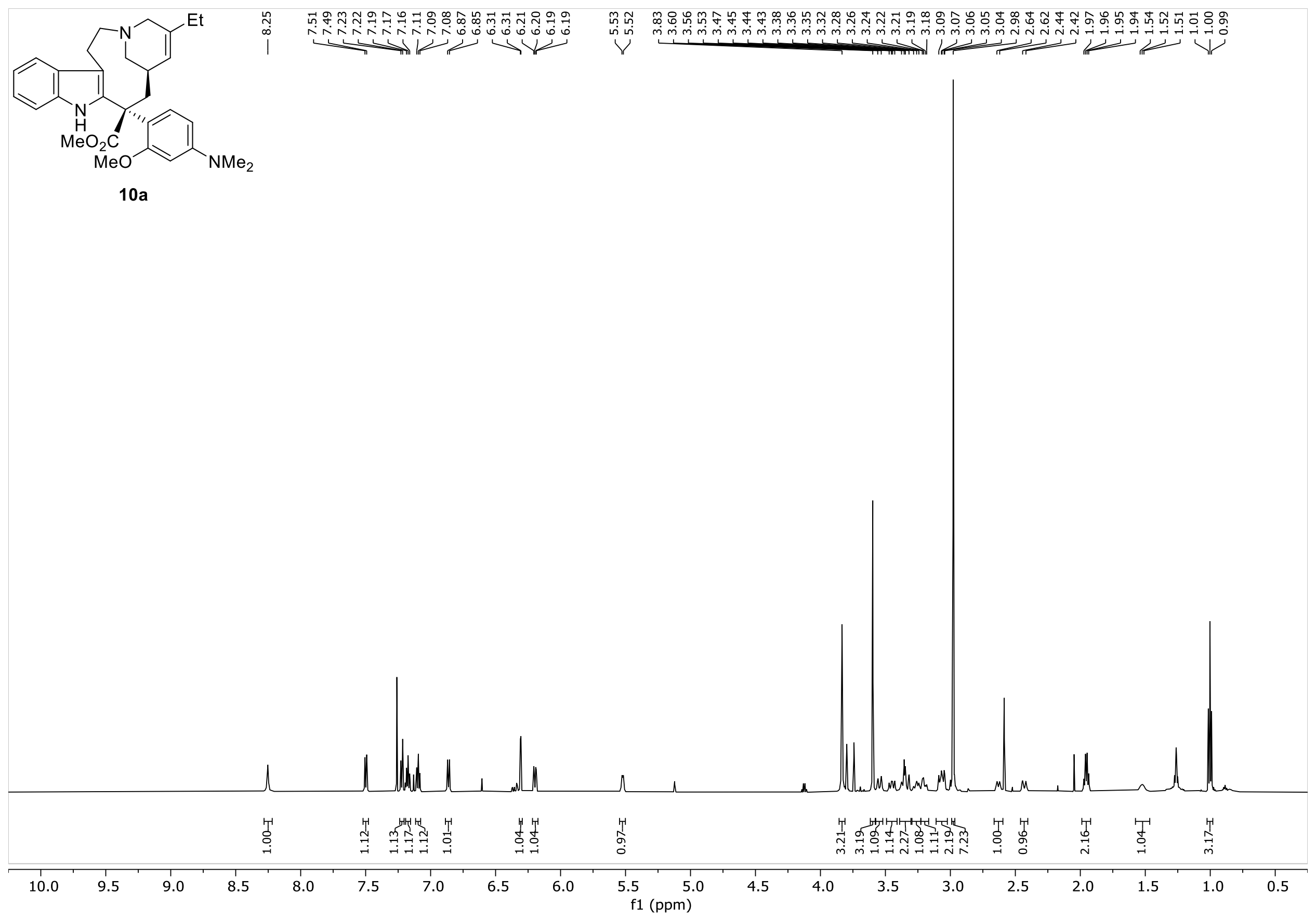




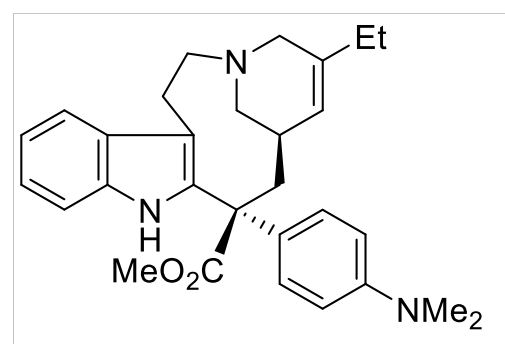

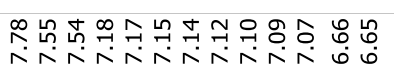

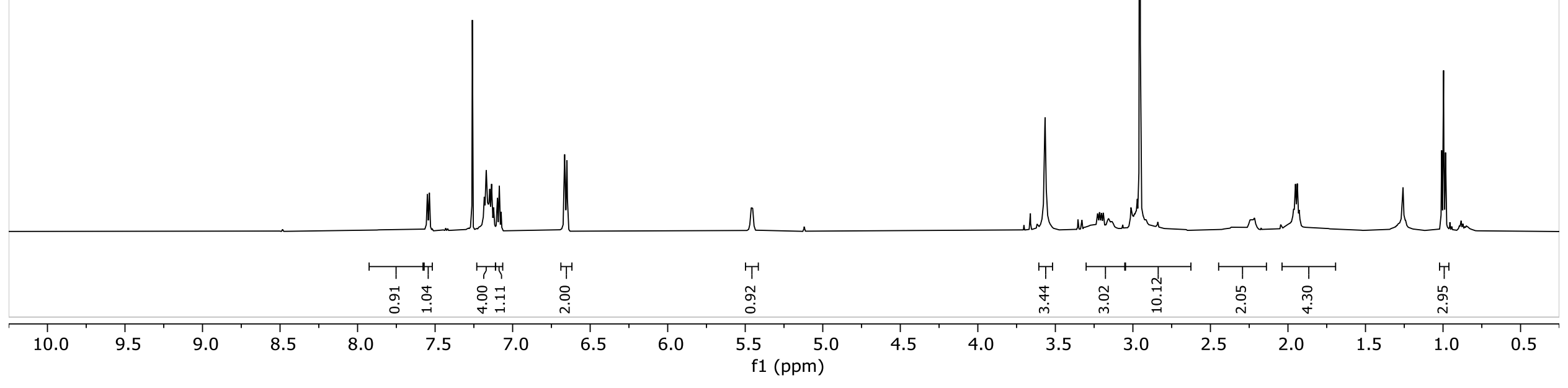




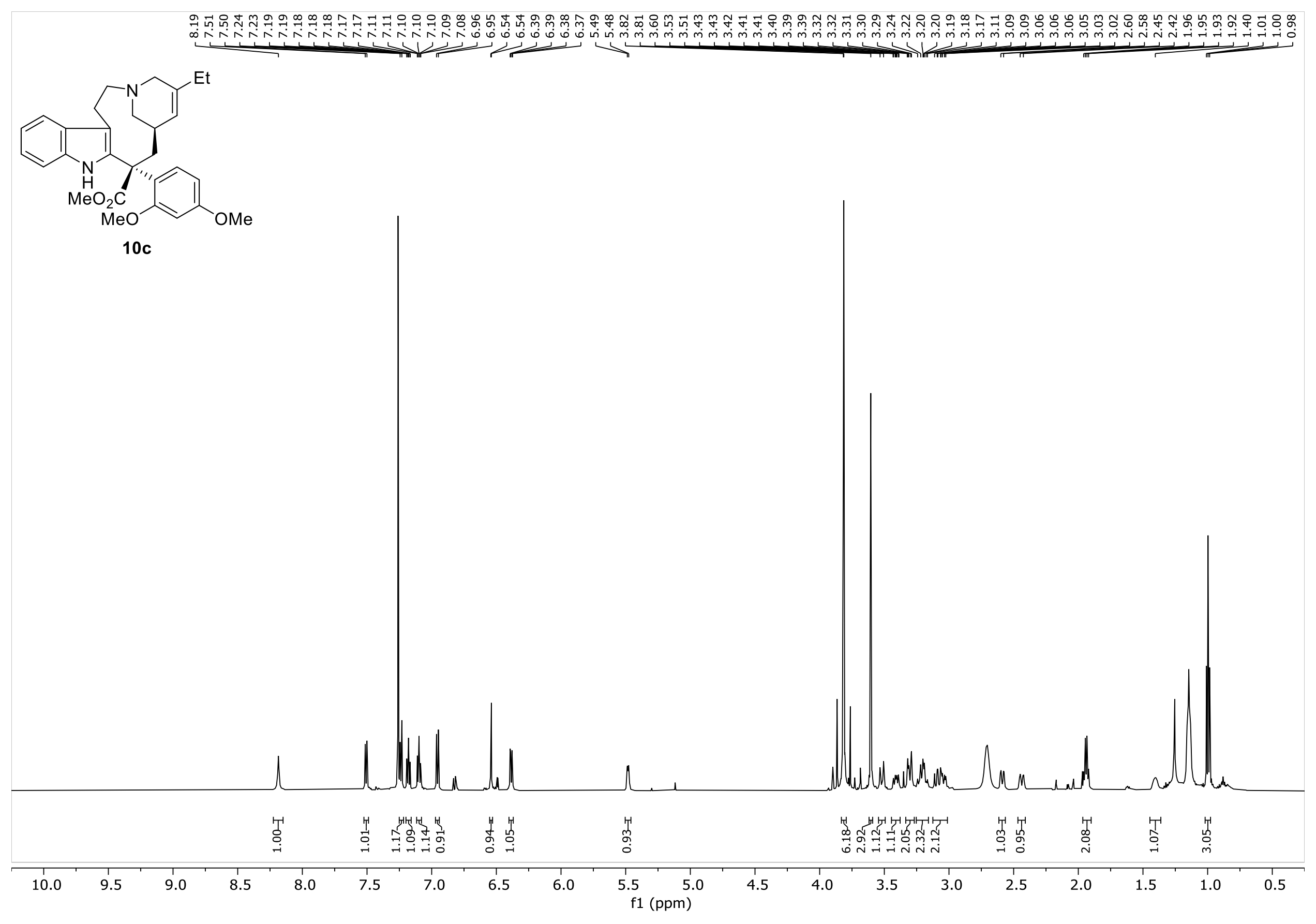




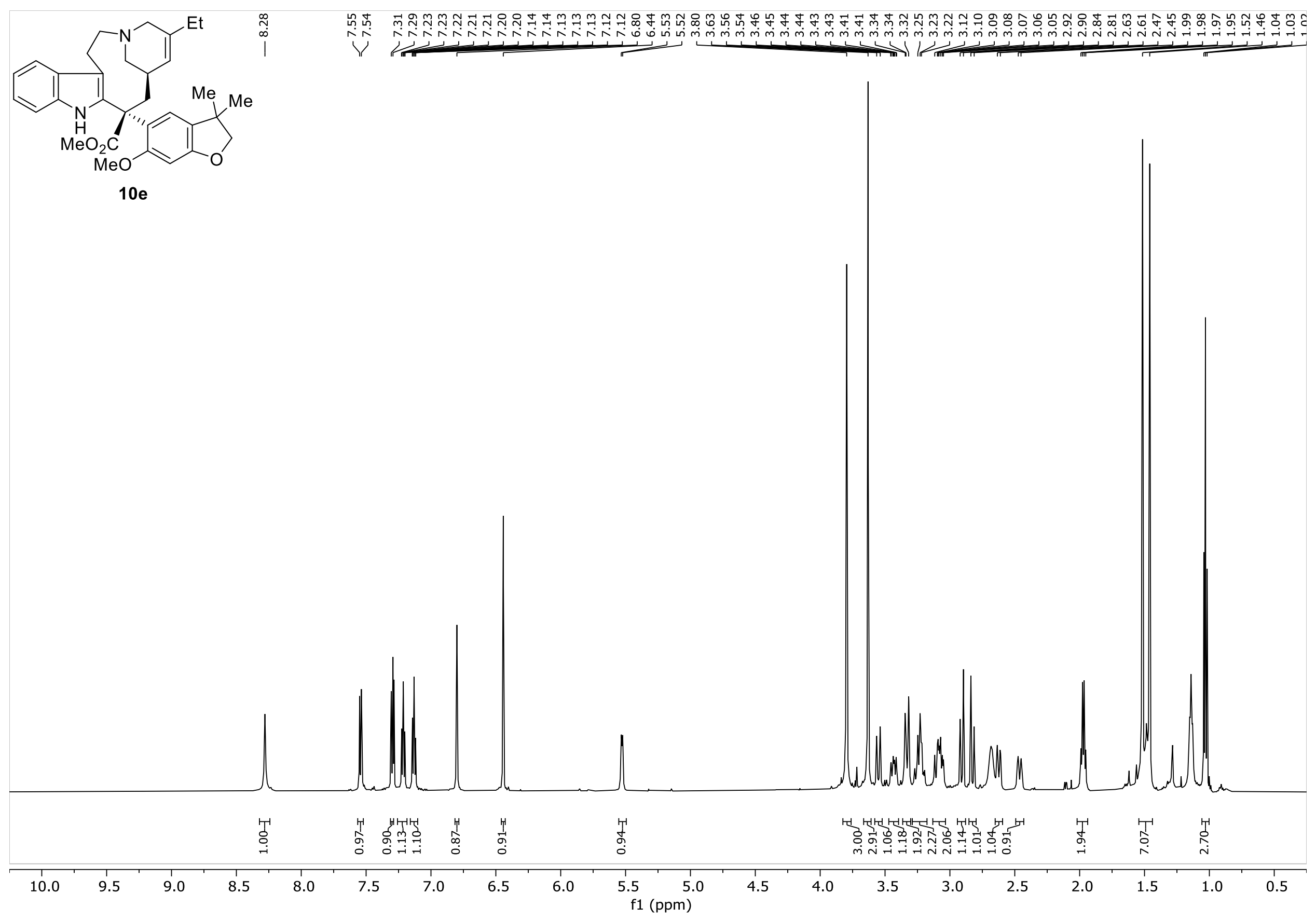




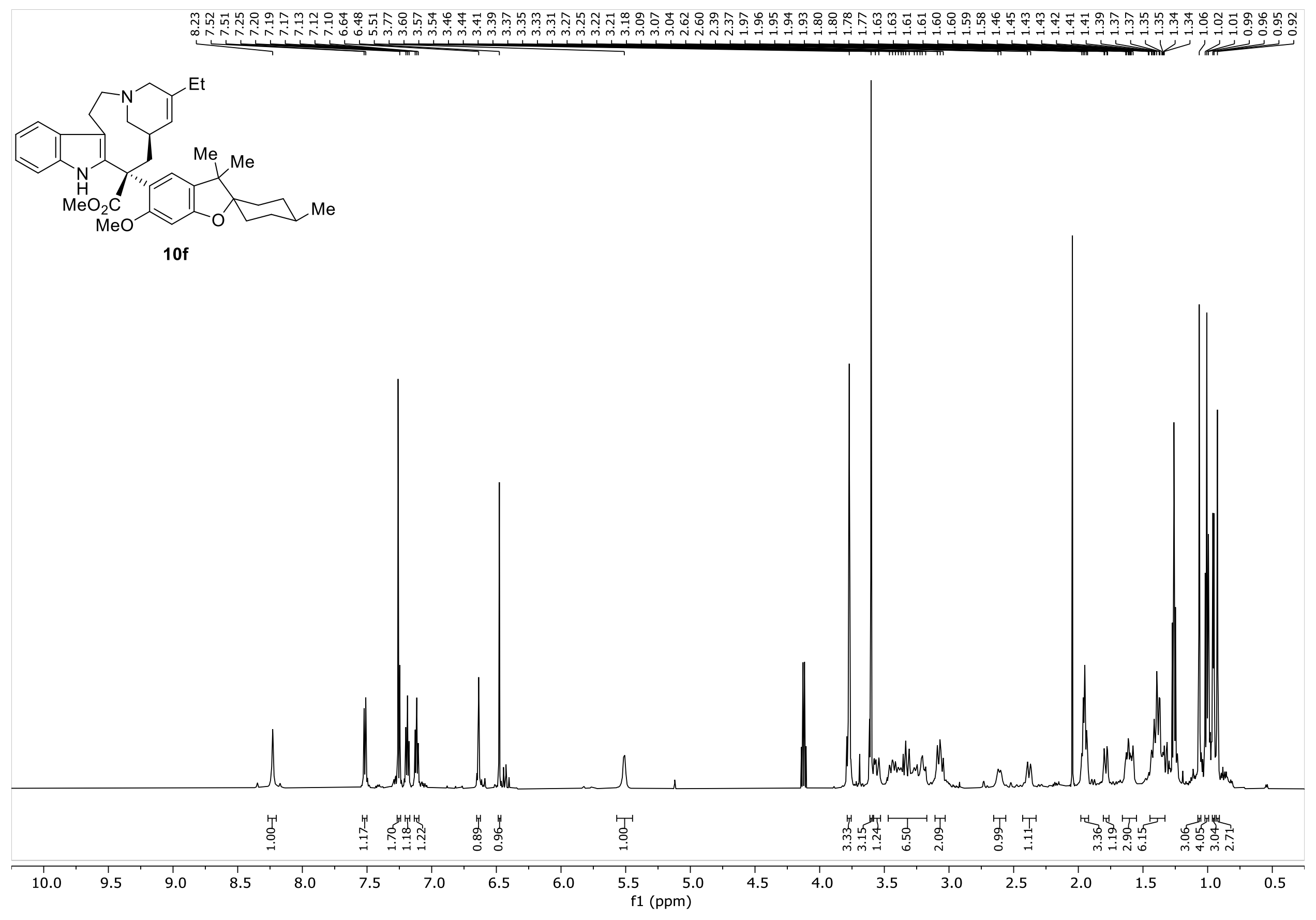




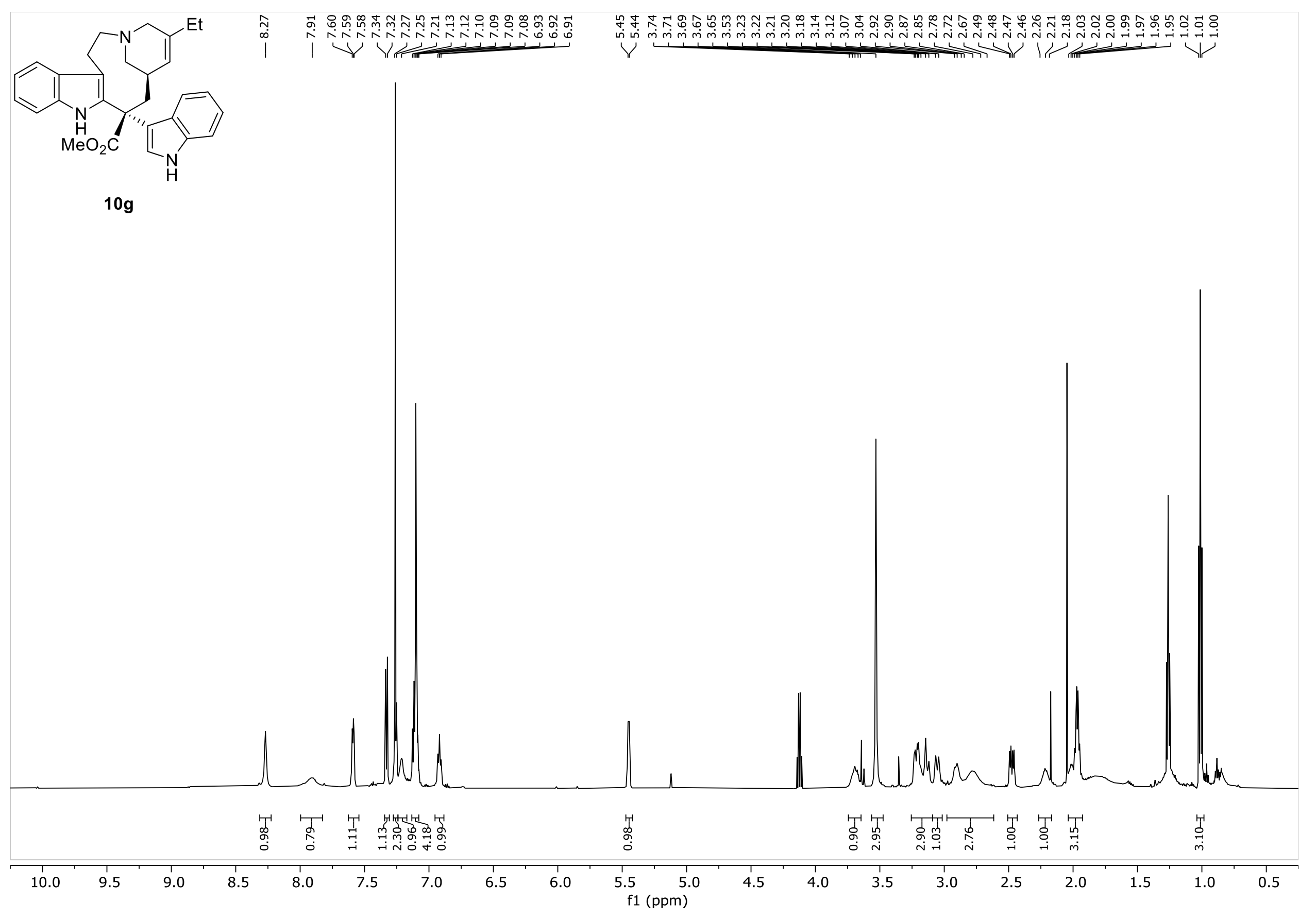




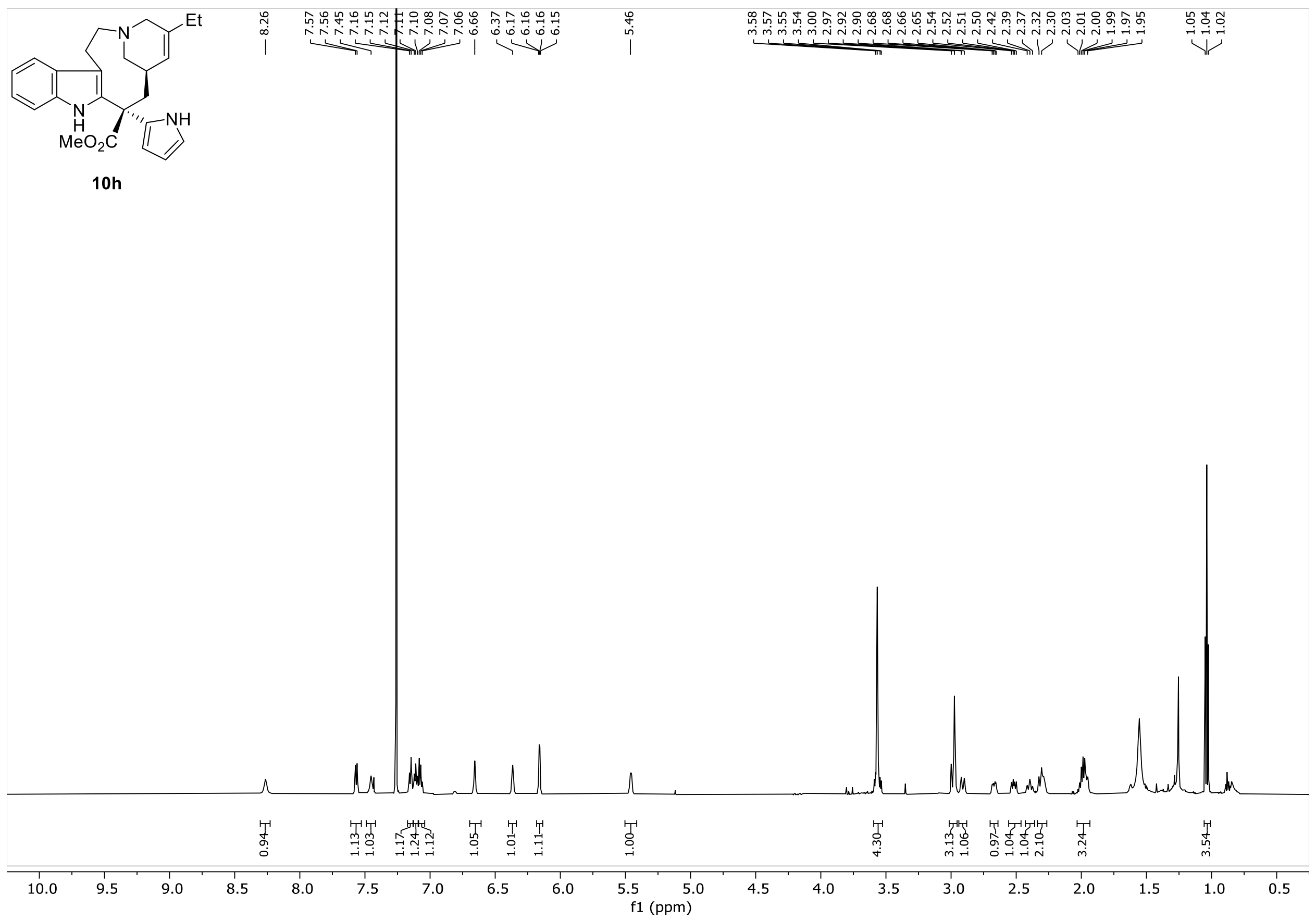




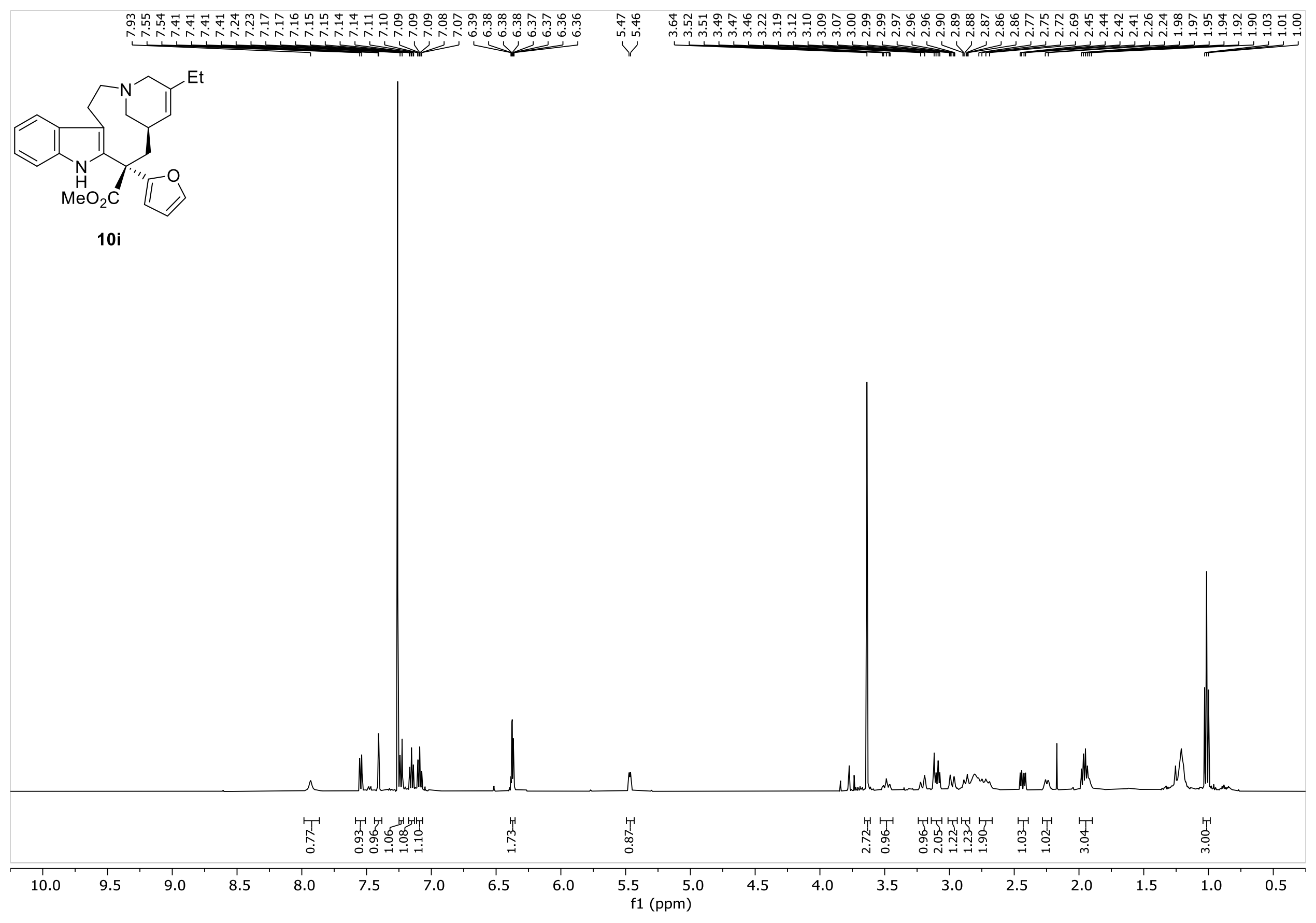




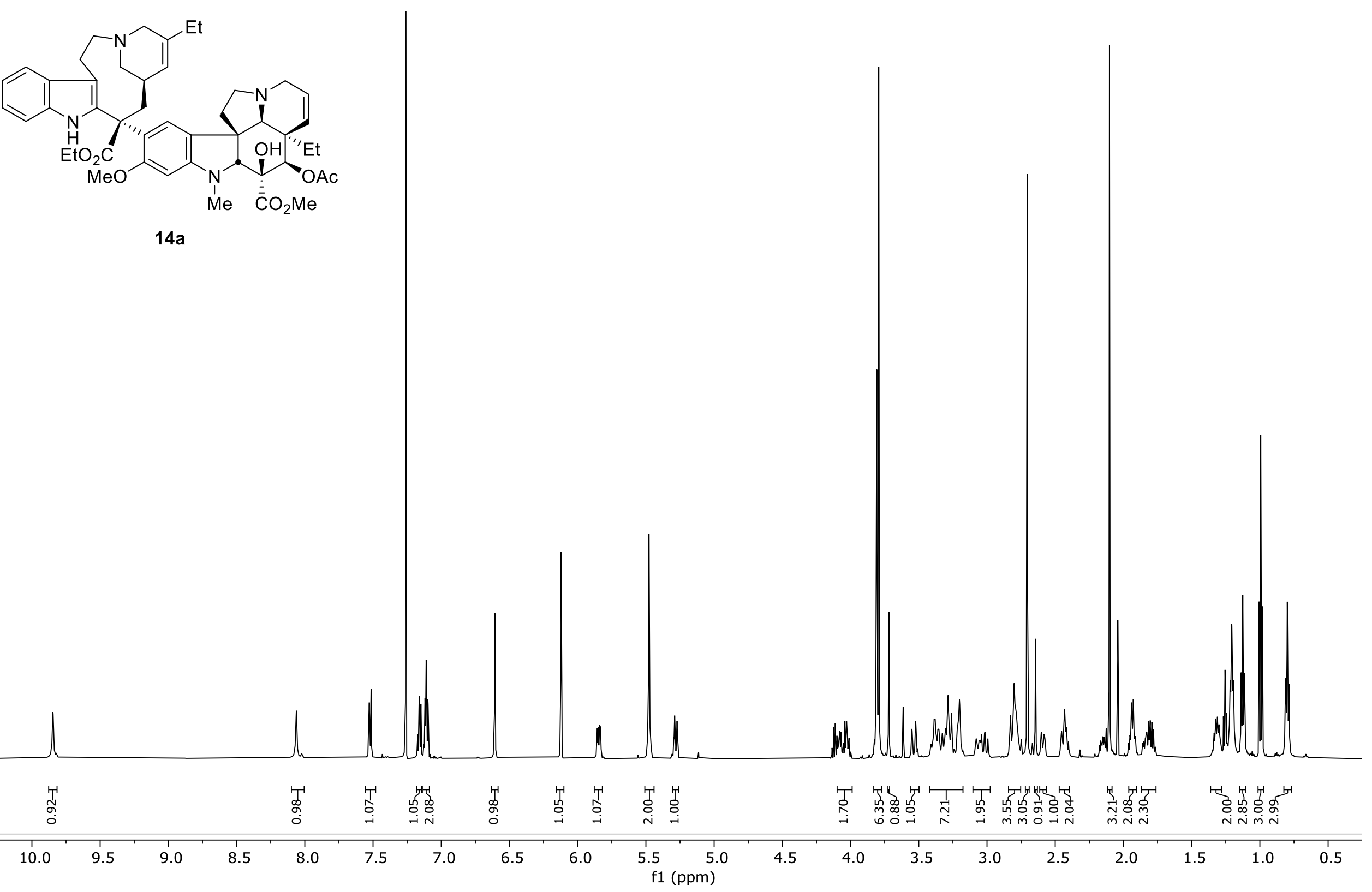




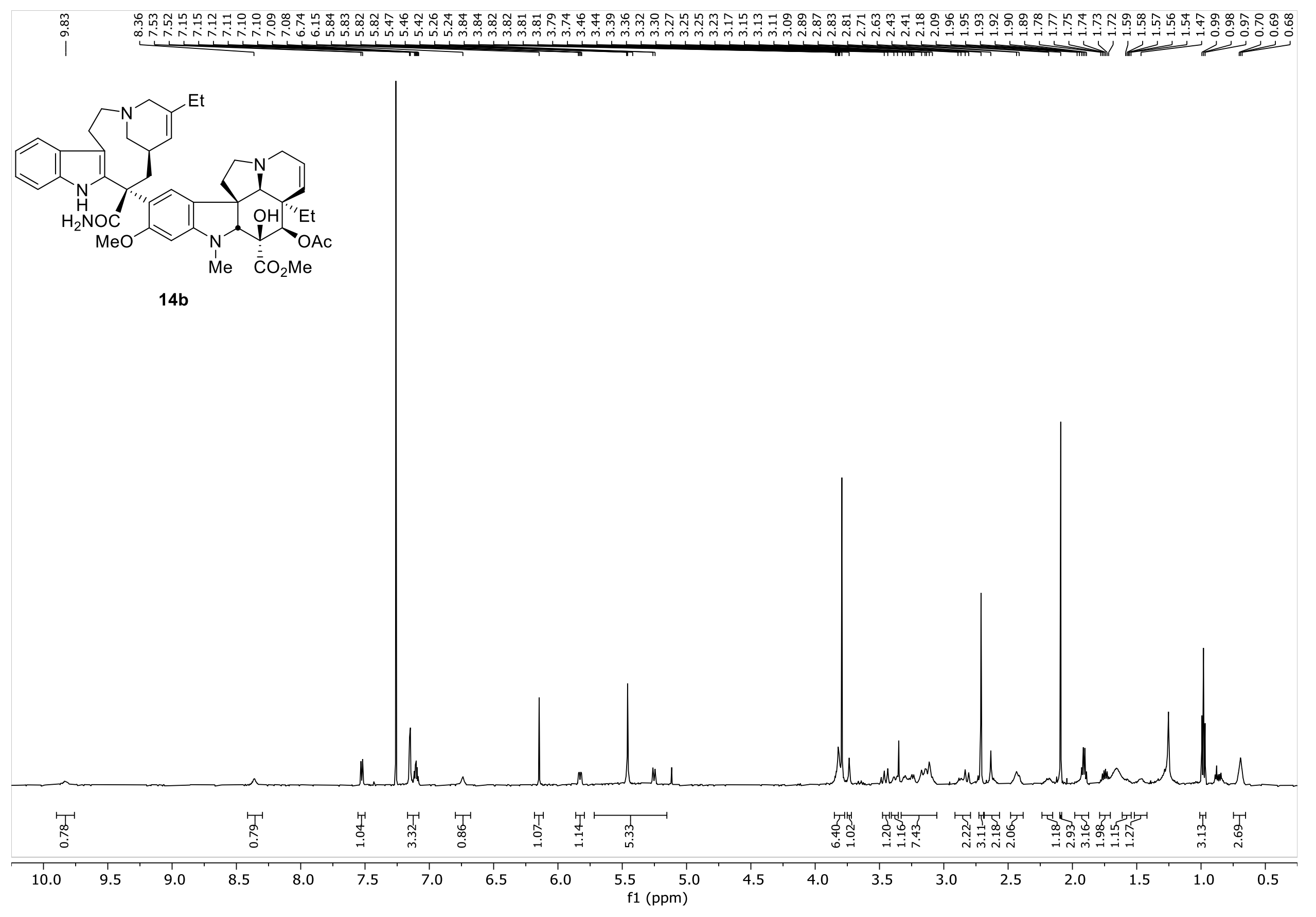




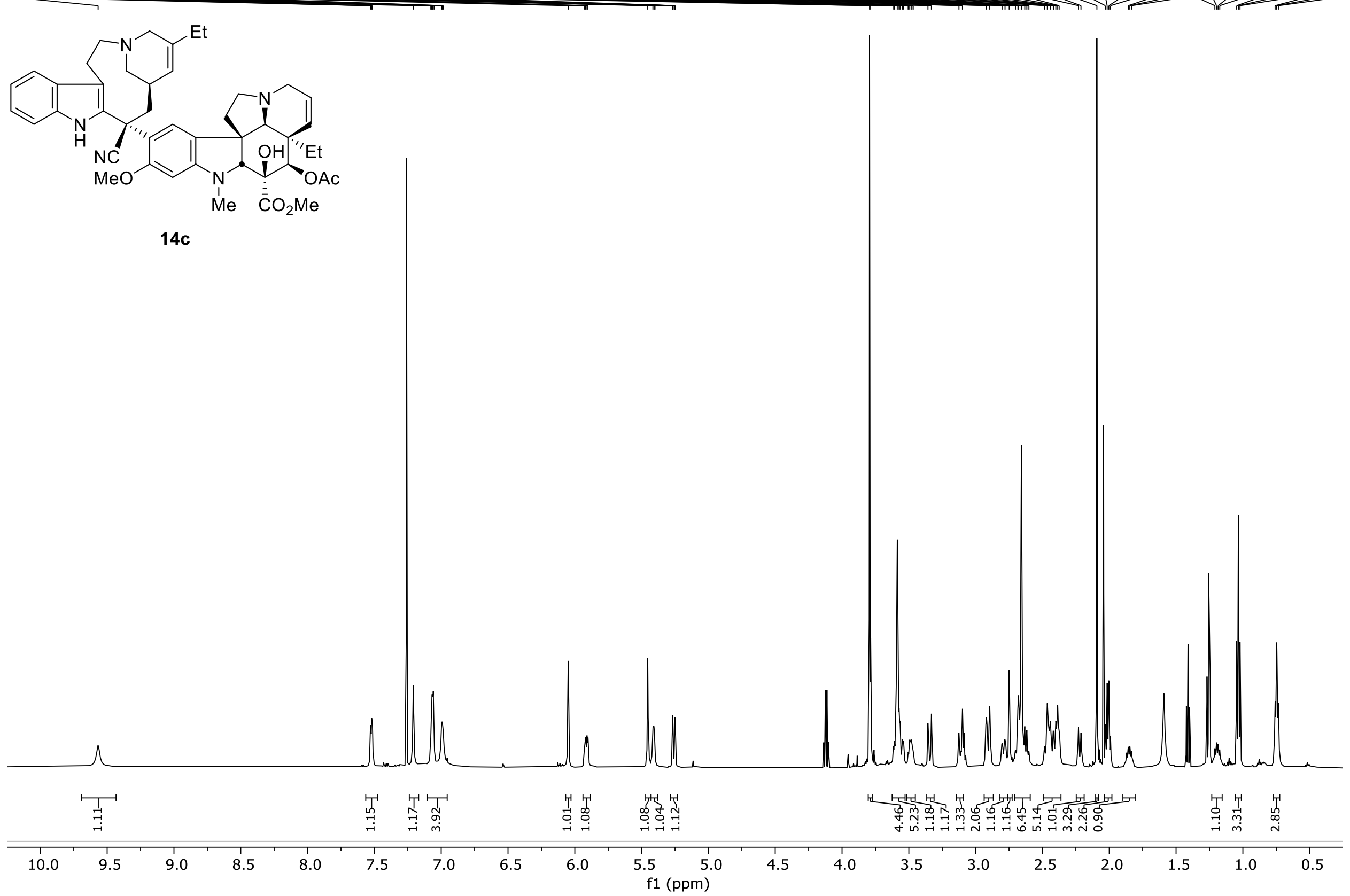




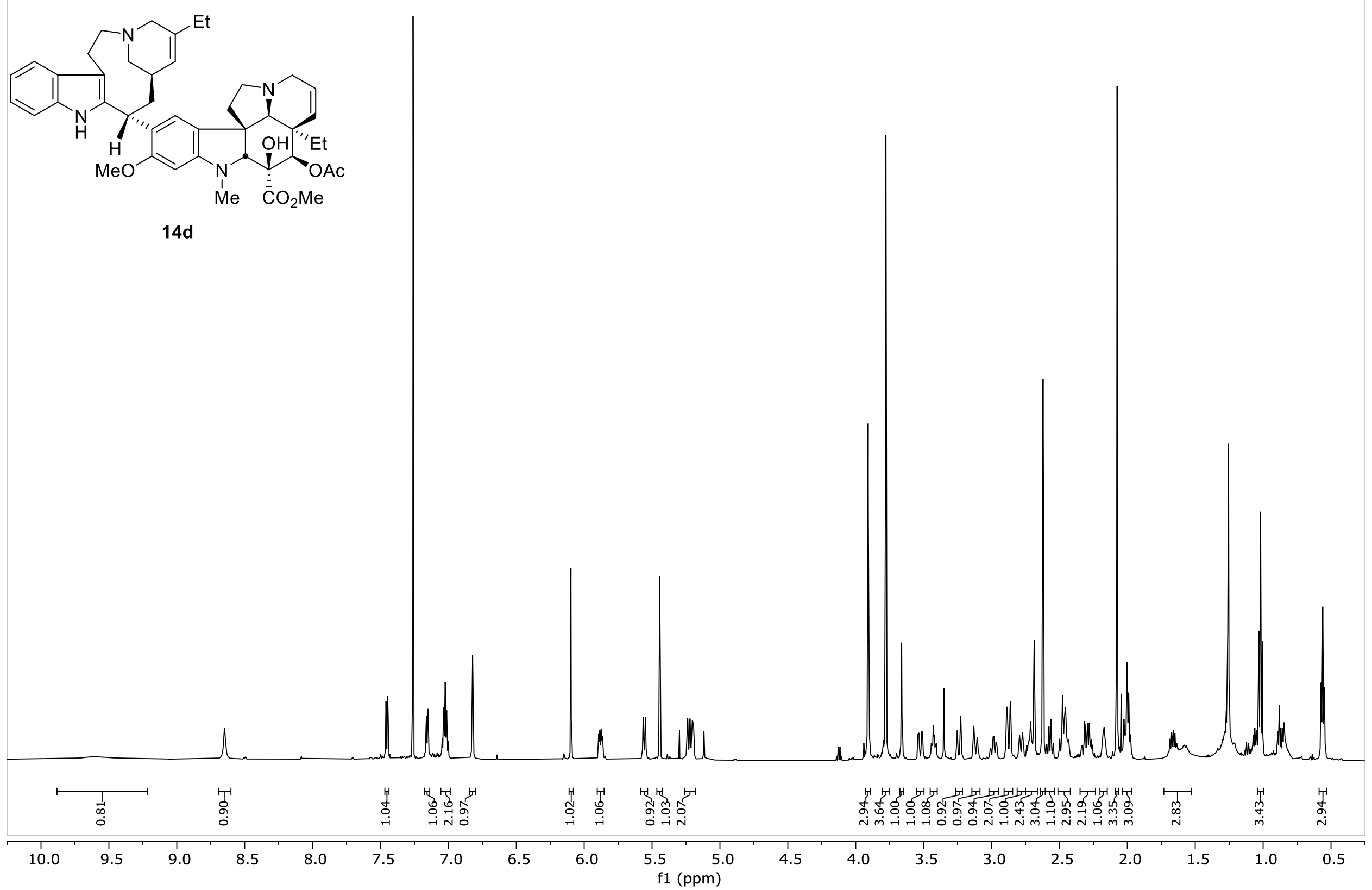




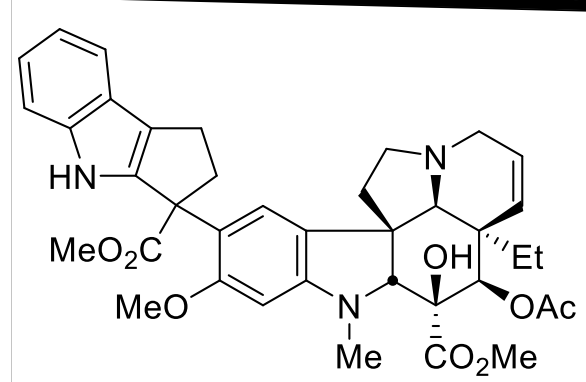

3'S-15

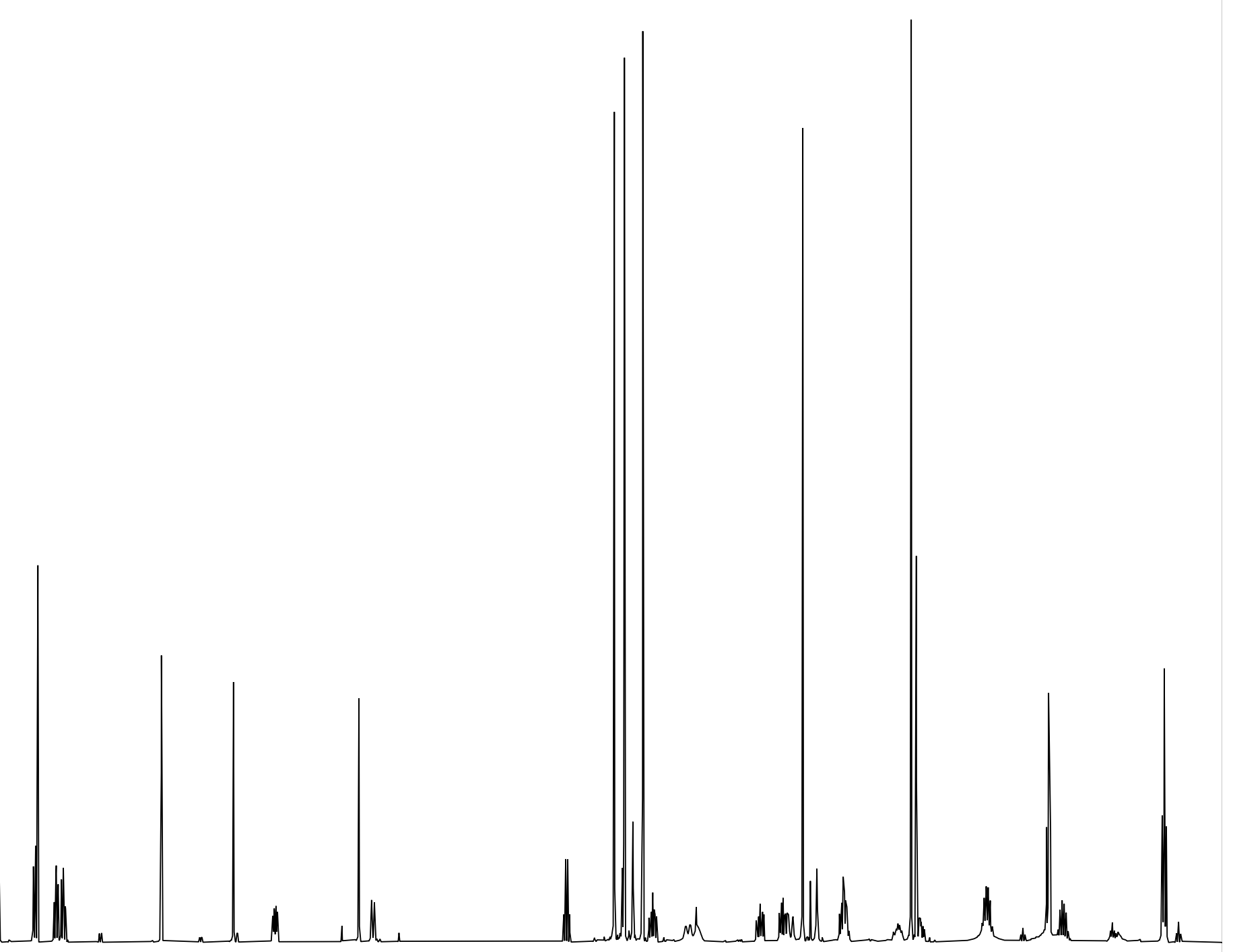

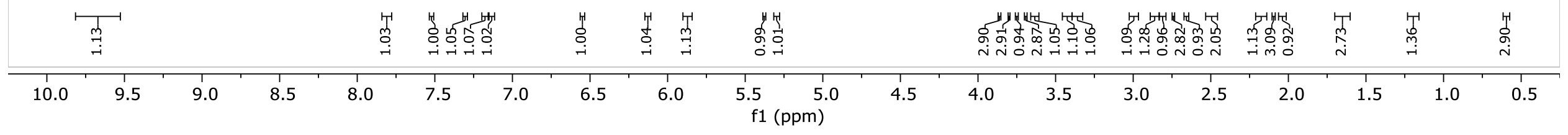




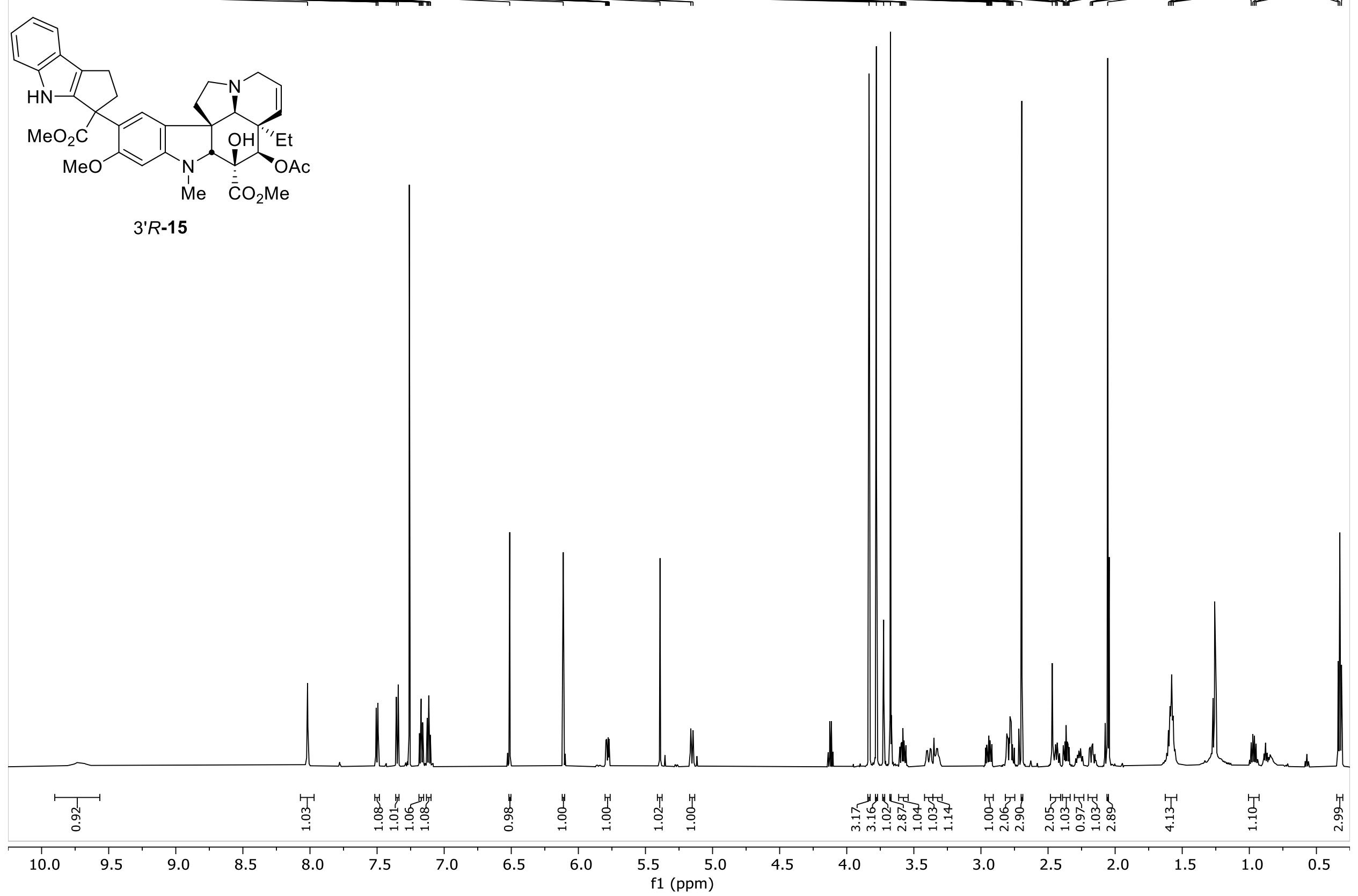




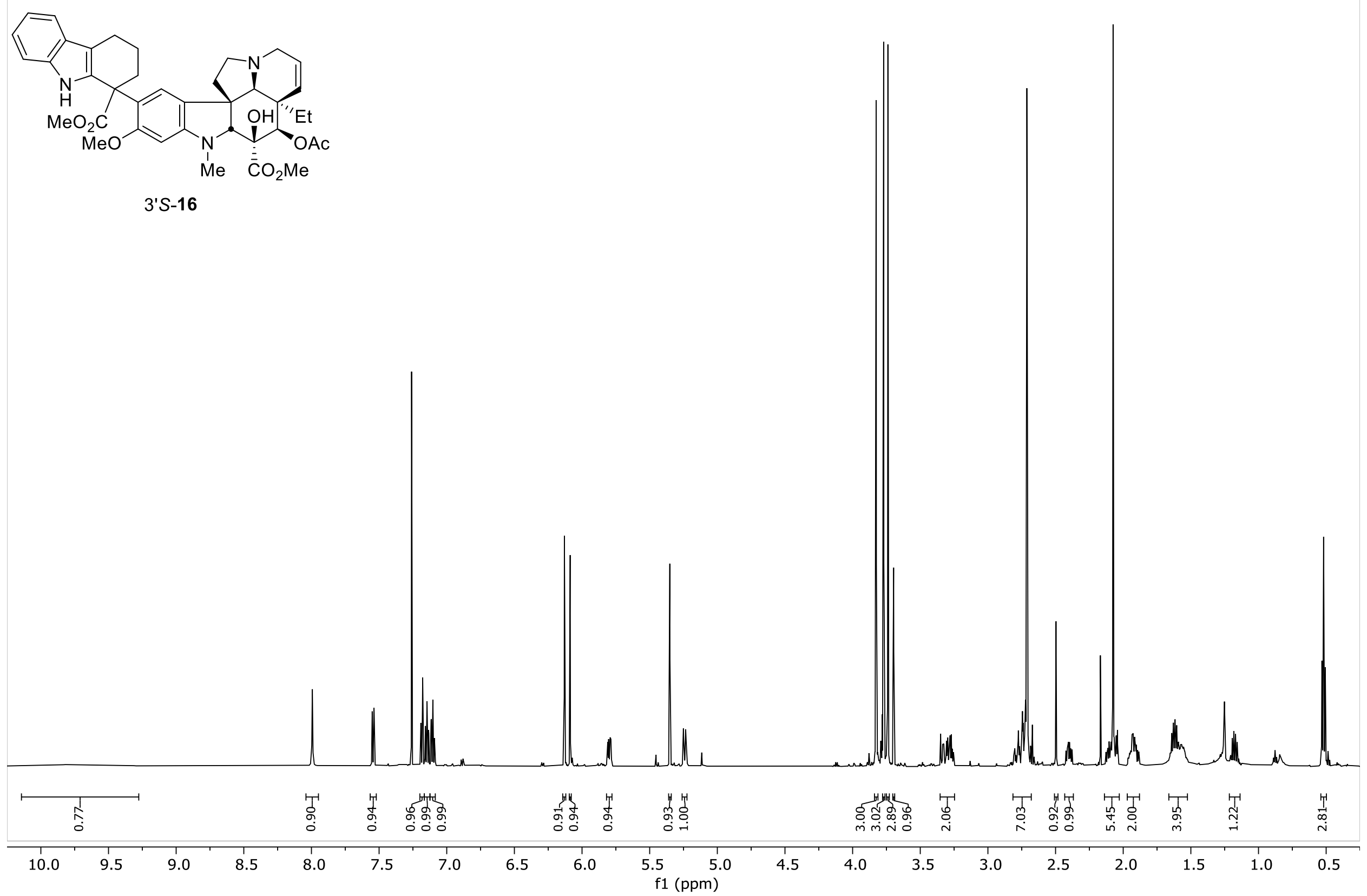




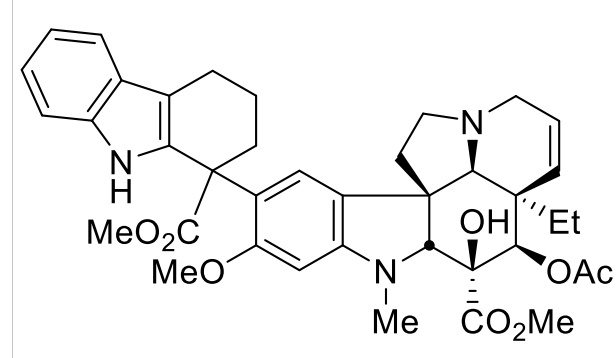

$3^{\prime} R-16$

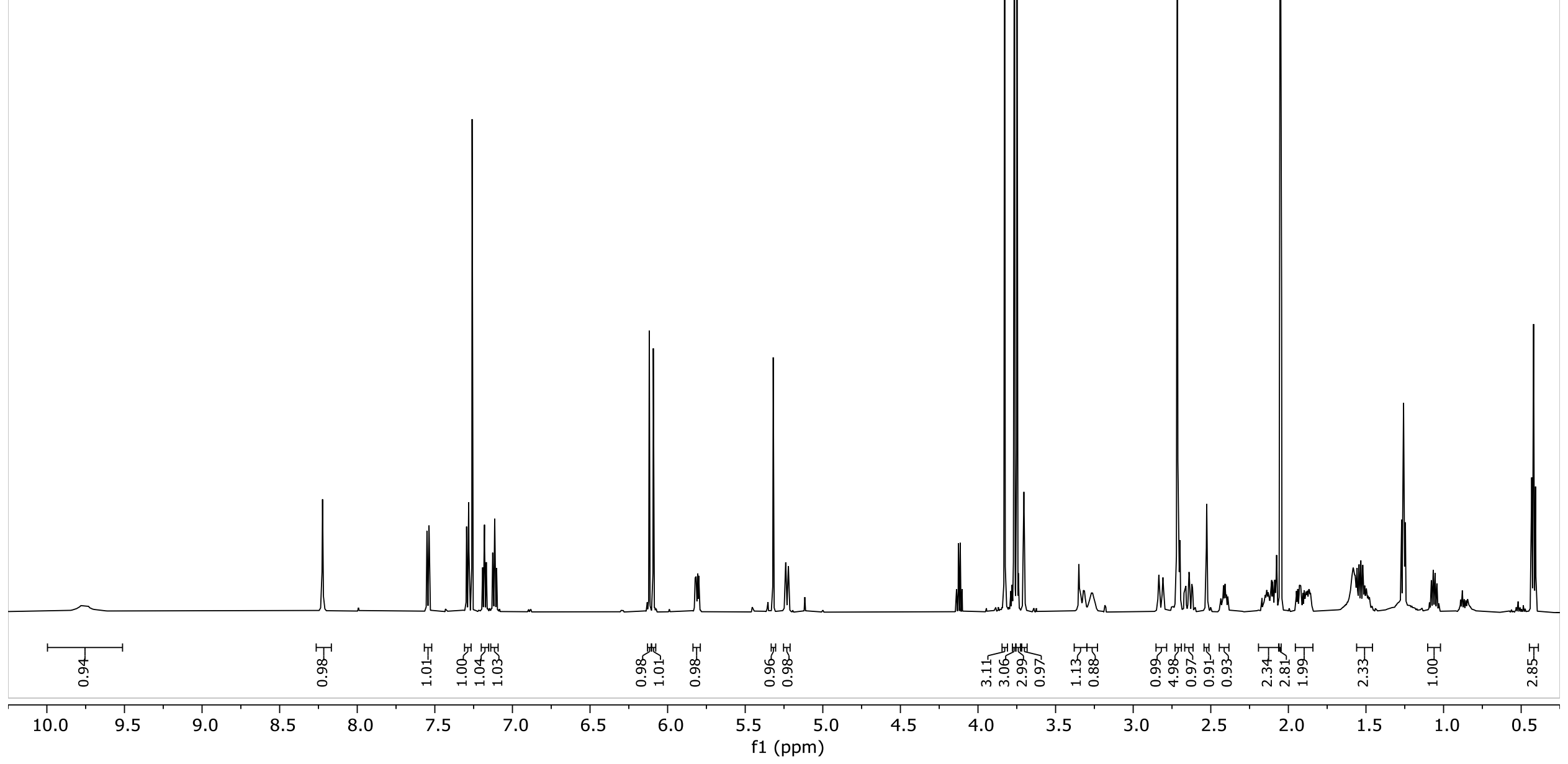




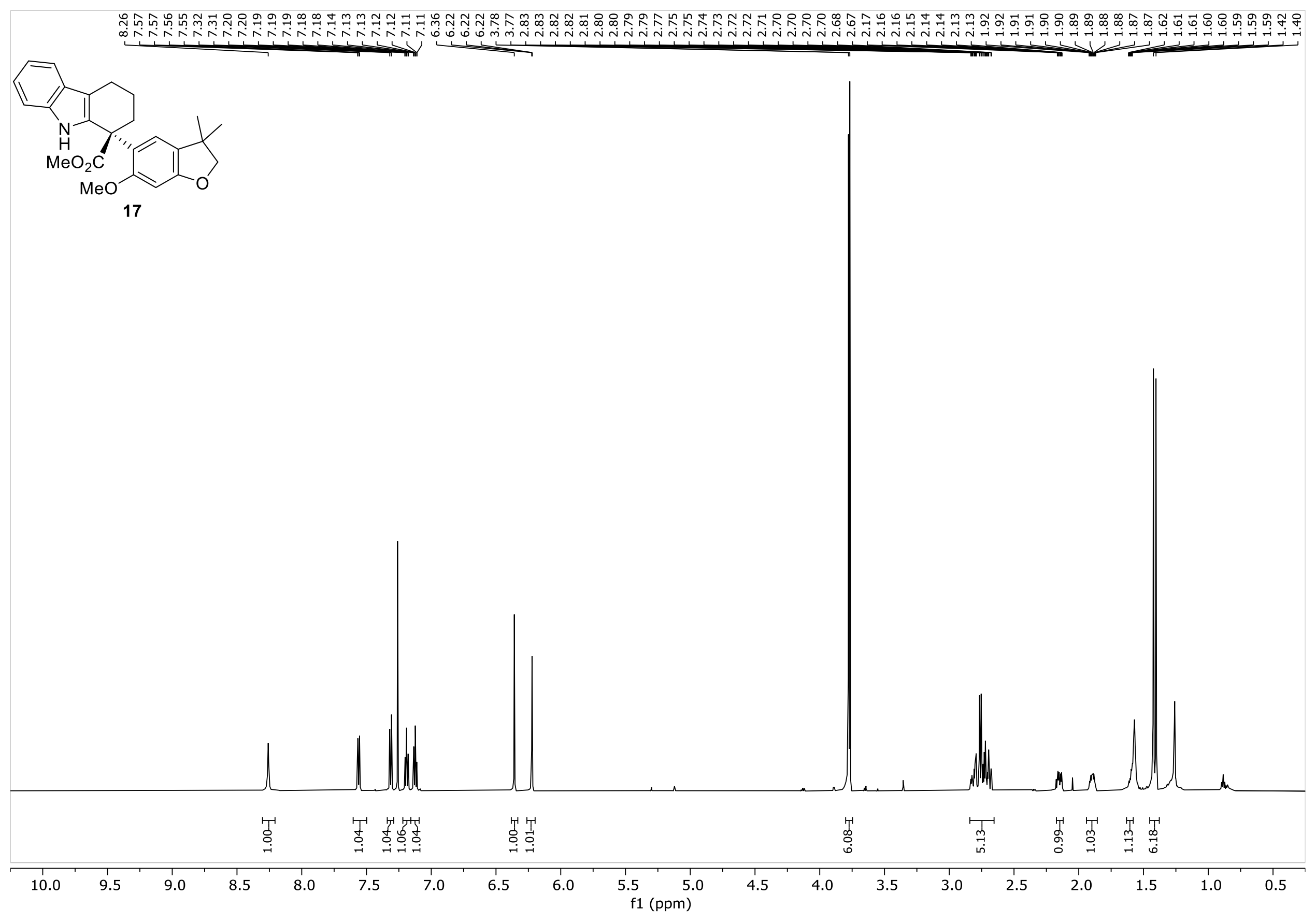




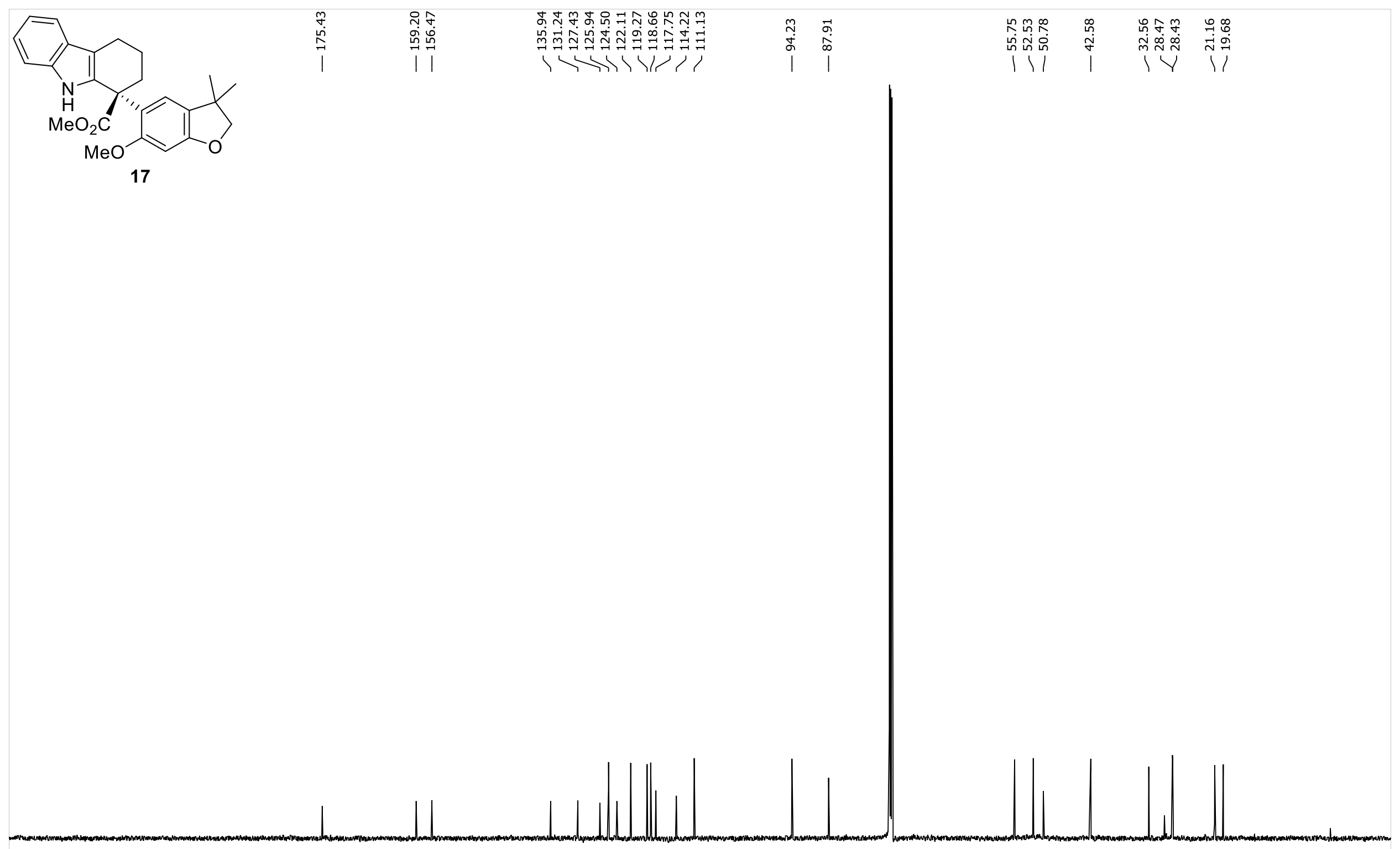

220

$210 \quad 200$

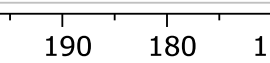

$170 \quad 160$

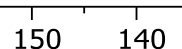

130

$20 \begin{gathered}110 \\ f 1(p p m)\end{gathered}$ 


\section{X-Ray Crystallography Data for 3'S-15 (CCDC 1947271)}

Single crystal X-ray diffraction studies were carried out on a Bruker SMART APEX II CCD diffractometer equipped with $\mathrm{Cu} \mathrm{K}_{\alpha}$ radiation $(\lambda=1.54178)$. Crystals of the subject compound were grown from $\mathrm{MeOH} /$ pentanes by vapor diffusion. A $0.180 \times 0.170 \times 0.125 \mathrm{~mm}$ light yellow block was mounted on a Cryoloop with Paratone oil.

Data were collected in a nitrogen gas stream at 100(2) $\mathrm{K}$ using $\phi$ and $\omega$ scans. Crystal-to-detector distance was 40 $\mathrm{mm}$ using exposure times $1,2,4$ and $8 \mathrm{~s}$ (depending on the 2theta range) with a scan width of $1.25^{\circ}$. Data collection was $100.0 \%$ complete to $67.679^{\circ}$ in $\theta$. A total of 58779 reflections were collected covering the indices, $-12<=\mathrm{h}<=13$, $18<=k<=18,-13<=\mid<=13.6707$ reflections were found to be symmetry independent, with a $R_{\text {int }}$ of 0.0242 . Indexing and unit cell refinement indicated a Primitive, Monoclinic lattice. The space group was found to be $\mathbf{P} \mathbf{2}_{\mathbf{1}}$. The data were integrated using the Bruker SAINT Software program and scaled using the SADABS software program. Solution by direct methods (SHELXT) produced a complete phasing model.

All nonhydrogen atoms were refined anisotropically by full-matrix least-squares (SHELXL-2014). All carbon bonded hydrogen atoms were placed using a riding model. Their positions were constrained relative to their parent atom using the appropriate HFIX command in SHELXL-2014.

Crystallographic data are summarized in Table $\mathbf{S 1 .}$

Notes: Excellent data and refinement, methanol solvate

Minor disorder on $\mathrm{CH}_{2}-\mathrm{CH}_{2}$ bridge and neighboring atoms.

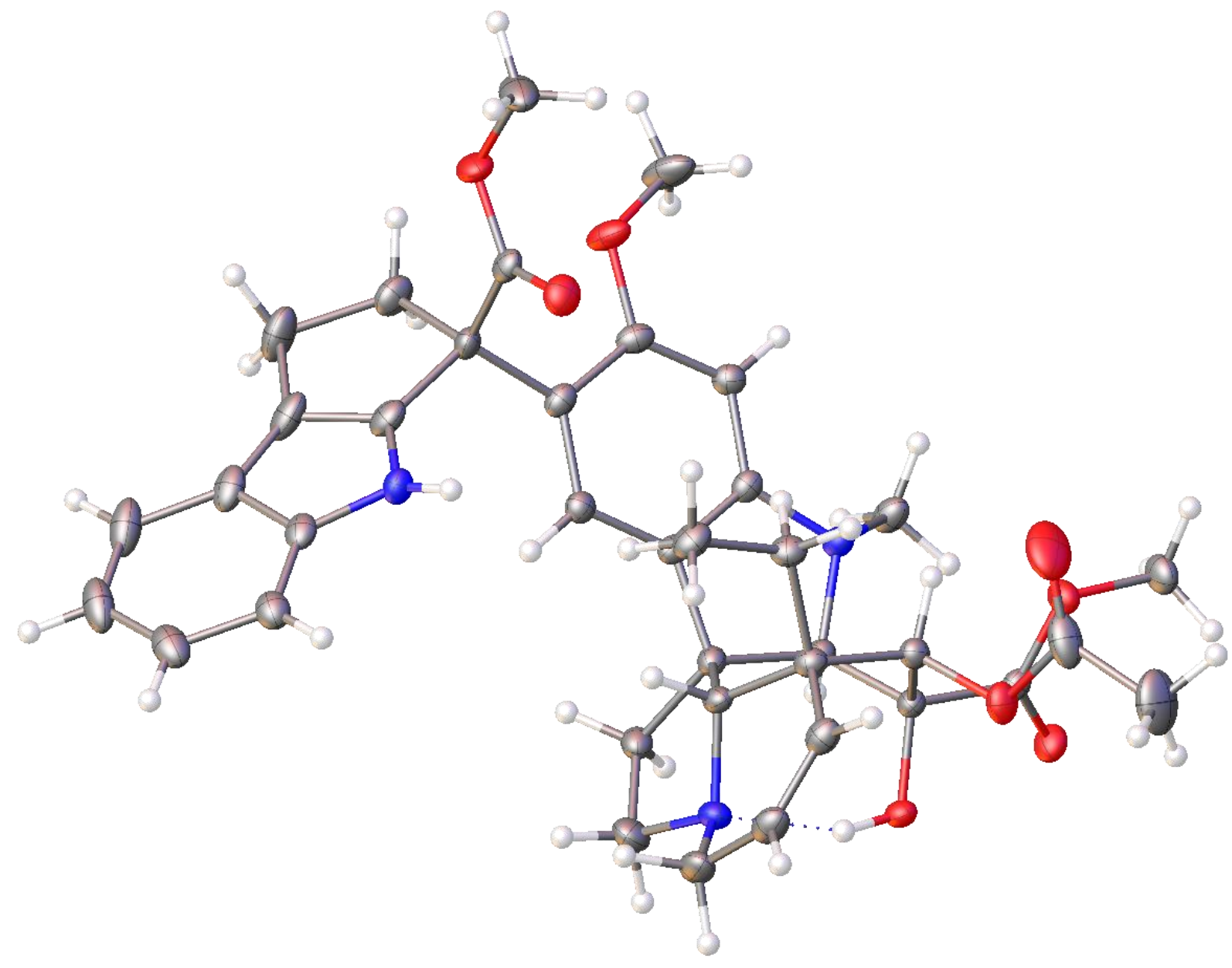


Table S1. Crystal data and structure refinement for 3'S-15.

Molecular formula

Formula weight

Temperature

Wavelength

Crystal system

Space group

Unit cell dimensions

Volume

Z

Density (calculated)

Absorption coefficient

$\mathrm{F}(000)$

Crystal size

Crystal color, habit

Theta range for data collection

Index ranges

Reflections collected

Independent reflections

Completeness to theta $=67.679^{\circ}$

Absorption correction

Max. and min. transmission

Refinement method

Data / restraints / parameters

Goodness-of-fit on $\mathrm{F}^{2}$

Final $R$ indices [ $1>2$ sigma(I)]

$R$ indices (all data)

Absolute structure parameter

Largest diff. peak and hole

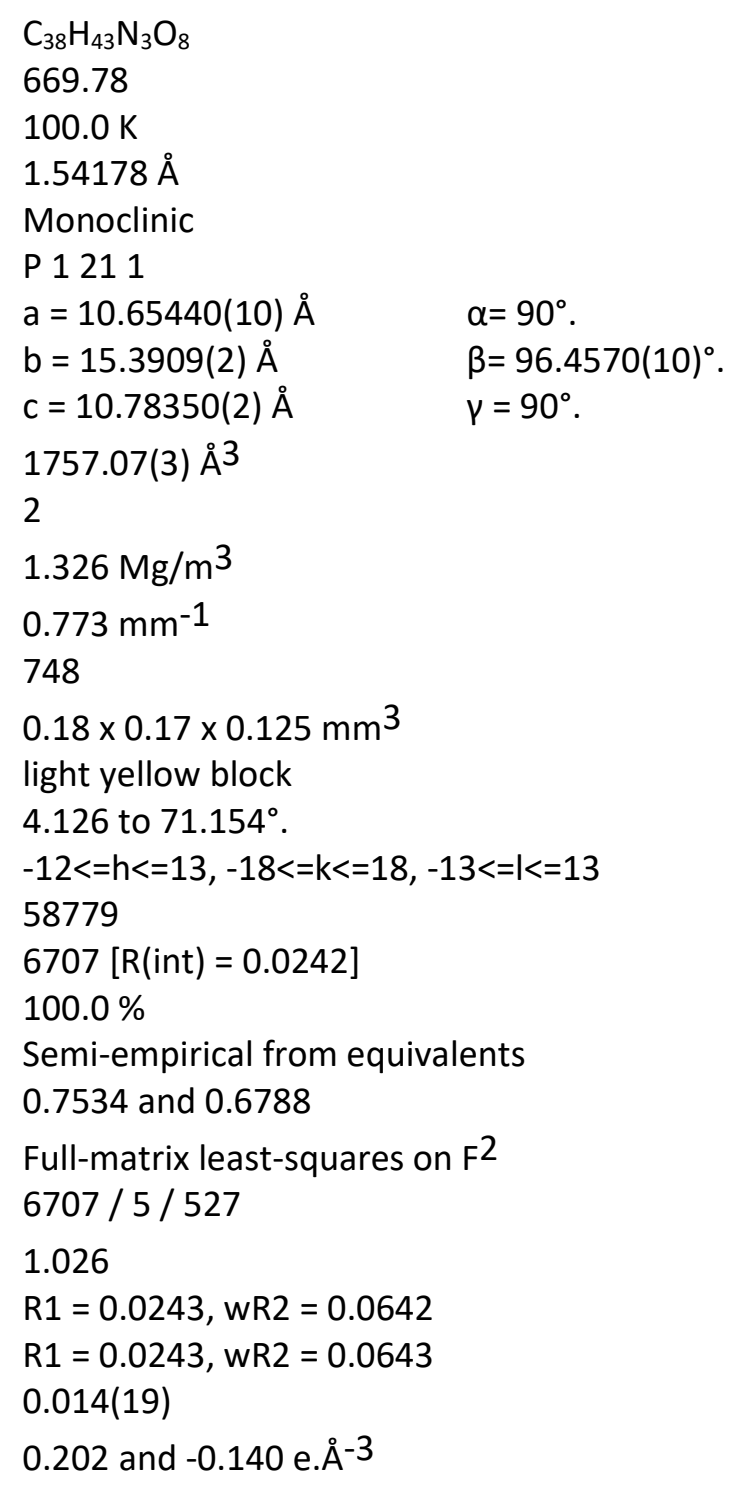




\section{X-Ray Crystallography Data for 3'R-16 (CCDC 1947270)}

Single crystal X-ray diffraction studies were carried out on a Bruker SMART APEX II CCD diffractometer equipped with $\mathrm{Cu} \mathrm{K} \mathrm{K}_{\alpha}$ radiation $(\lambda=1.54178)$. Crystals of the subject compound were grown from benzene/pentanes by vapor diffusion. A $0.125 \times 0.040 \times 0.015 \mathrm{~mm}$ colorless irregular crystal was mounted on a Cryoloop with Paratone oil.

Data were collected in a nitrogen gas stream at 100(2) $\mathrm{K}$ using $\phi$ and $\omega$ scans. Crystal-to-detector distance was 45 $\mathrm{mm}$ using exposure times 15,40 and $60 \mathrm{~s}$ (depending on the 2theta range) with a scan width of $1.25^{\circ}$. Data collection was $97.8 \%$ complete to $67.500^{\circ}$ in $\theta$. A total of 54822 reflections were collected covering the indices, $-9<=\mathrm{h}<=9,-33<=\mathrm{k}<=23$, $37<=\mid<=30.14071$ reflections were found to be symmetry independent, with a $R_{\text {int }}$ of 0.1007 . Indexing and unit cell refinement indicated a Primitive, Orthorhombic lattice. The space group was found to be $\mathbf{P} \mathbf{2}_{\mathbf{1}} \mathbf{2}_{\mathbf{1}} \mathbf{2}_{\mathbf{1}}$. The data were integrated using the Bruker SAINT Software program and scaled using the SADABS software program. Solution by direct methods (SHELXT) produced a complete phasing model.

All nonhydrogen atoms were refined anisotropically by full-matrix least-squares (SHELXL-2014). All carbon bonded hydrogen atoms were placed using a riding model. Their positions were constrained relative to their parent atom using the appropriate HFIX command in SHELXL-2014.

Crystallographic data are summarized in Table S2.

Notes: Two molecules in asymmetric unit.

Disorder benzene solvent molecules have been removed using SQUEEZE procedure.

Limited data quality due to crystal size/quality, decent refinement

Refinement conclusive with "Absolute structure parameter $0.07(13)$ "

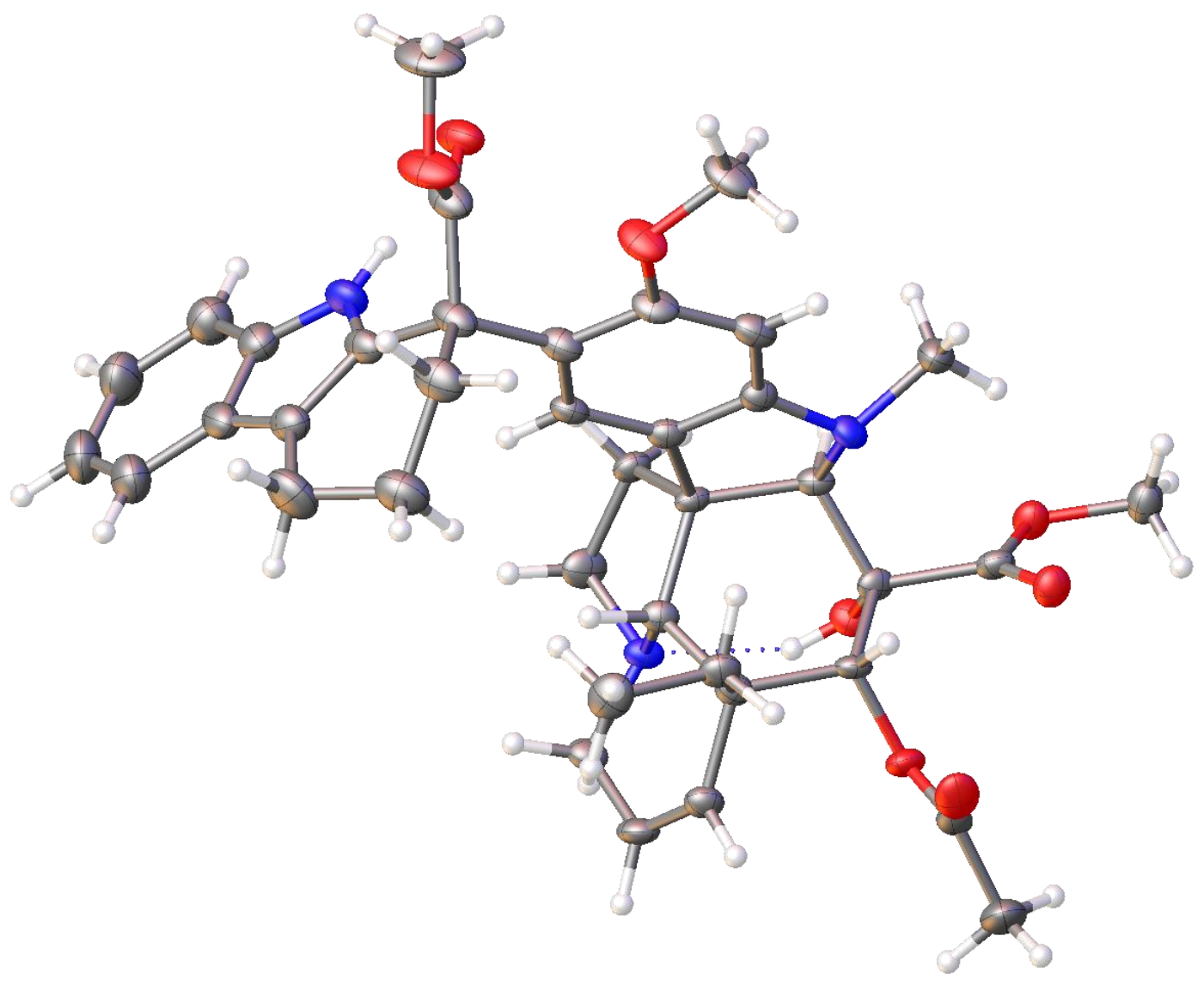


Table S2. Crystal data and structure refinement for $3^{\prime} R-16$.

Molecular formula

Formula weight

Temperature

Wavelength

Crystal system

Space group

Unit cell dimensions

Volume

Z

Density (calculated)

Absorption coefficient

$\mathrm{F}(000)$

Crystal size

Crystal color, habit

Theta range for data collection

Index ranges

Reflections collected

Independent reflections

Completeness to theta $=67.679^{\circ}$

Absorption correction

Max. and min. transmission

Refinement method

Data / restraints / parameters

Goodness-of-fit on $F^{2}$

Final $R$ indices [ $>2$ sigma $(I)]$

$R$ indices (all data)

Absolute structure parameter

Extinction coefficient

Largest diff. peak and hole
$\mathrm{C}_{39} \mathrm{H}_{45} \mathrm{~N}_{3} \mathrm{O}_{8}$

683.80

$100.0 \mathrm{~K}$

$1.54178 \AA$

Monoclinic

$\mathrm{P} 2{ }_{1} 2{ }_{1} 2_{1}$

$\mathrm{a}=8.1852(2) \AA$

$\alpha=90^{\circ}$.

$\mathrm{b}=30.4869(7) \AA$

$\beta=90^{\circ}$.

$c=31.5036(8) \AA \quad \gamma=90^{\circ}$.

$7861.5(3) \AA^{3}$

8

$1.155 \mathrm{Mg} / \mathrm{m}^{3}$

$0.660 \mathrm{~mm}^{-1}$

2912

$0.125 \times 0.04 \times 0.015 \mathrm{~mm}^{3}$

colorless irregular

2.017 to $68.304^{\circ}$.

$-9<=\mathrm{h}<=9,-33<=\mathrm{k}<=23,-37<=\mathrm{k}<=30$

54822

$14071[R($ int $)=0.1007]$

$97.8 \%$

Semi-empirical from equivalents

0.7515 and 0.6943

Full-matrix least-squares on $\mathrm{F}^{2}$

14071 / 0 / 915

0.963

$\mathrm{R} 1=0.0506, \mathrm{wR} 2=0.1043$

$\mathrm{R} 1=0.0813, \mathrm{wR} 2=0.1161$

$0.07(13)$

$\mathrm{n} / \mathrm{a}$

0.320 and -0.208 e. $\AA^{-3}$ 